\title{
How Spike Generation Mechanisms Determine the Neuronal Response to Fluctuating Inputs
}

\author{
Nicolas Fourcaud-Trocmé, David Hansel, Carl van Vreeswijk, and Nicolas Brunel \\ Centre National de la Recherche Scientifique Unité Mixte de Recherche 8119, Neurophysique et Physiologie du Système Moteur, Unité de Formation et de \\ Recherche Biomédicale, Université Paris 5 René Descartes, 75270 Paris Cedex 06, France
}

This study examines the ability of neurons to track temporally varying inputs, namely by investigating how the instantaneous firing rate of a neuron is modulated by a noisy input with a small sinusoidal component with frequency $(f)$. Using numerical simulations of conductance-based neurons and analytical calculations of one-variable nonlinear integrate-and-fire neurons, we characterized the dependence of this modulation on $f$. For sufficiently high noise, the neuron acts as a low-pass filter. The modulation amplitude is approximately constant for frequencies up to a cutoff frequency, $f_{c}$, after which it decays. The cutoff frequency increases almost linearly with the firing rate. For higher frequencies, the modulation amplitude decays as $C / f^{\alpha}$, where the power $\alpha$ depends on the spike initiation mechanism. For conductance-based models, $\alpha=1$, and the prefactor $C$ depends solely on the average firing rate and a spike "slope factor," which determines the sharpness of the spike initiation. These results are attributable to the fact that near threshold, the sodium activation variable can be approximated by an exponential function. Using this feature, we propose a simplified one-variable model, the "exponential integrate-and-fire neuron," as an approximation of a conductance-based model. We show that this model reproduces the dynamics of a simple conductance-based model extremely well. Our study shows how an intrinsic neuronal property (the characteristics of fast sodium channels) determines the speed with which neurons can track changes in input.

Key words: populations of spiking neurons; noise; dynamics; sodium channel; integrate-and-fire model; conductance-based model

\section{Introduction}

The input-output transformation performed by a neuron is classically characterized by its frequency-current $(f-I)$ relationship (McCormick et al., 1985; Powers and Binder, 2001). However, knowing of this relationship is not enough to predict neuronal responses to transients or time-dependent inputs, a problem that is, in general, particularly tricky because of the highly nonlinear nature of action potential generation. Nevertheless, if the temporal variations of the input are small enough, the dynamics are dominated by the linear response properties of the neuron. Therefore, the response of the neuron to an arbitrary input with weak temporal variations can be predicted if we can determine how its instantaneous firing rate is modulated by sinusoidal inputs.

The response of neurons to sinusoidal inputs has been investigated in various in vitro preparations, including the horseshoe crab Limulus polyphemus (Knight, 1972b), visual cortex (Carandini et al., 1996; Nowak et al., 1997), and vestibular nuclei (Ris et al., 2001; Sekirnjak and du Lac, 2002). Most of these studies restricted their analysis to low-frequency inputs $(<20 \mathrm{~Hz})$. Carandini et al. (1996) found that the response of regular-spiking cor-

Received July 28, 2003; revised 0ct. 15, 2003; accepted 0ct. 15, 2003. We thank Claude Meunier for a critical reading of a previous version of this manuscript.

Correspondence should be addressed to Nicolas Brunel, Centre National de la Recherche Scientifique, Neurophysique et Physiologie du Système Moteur, Université Paris René Descartes, 45 rue des Saints Pères, 75270 Paris Cedex 06, France. E-mail: nicolas.brunel@biomedicale.univ-paris5.fr.

Copyright $\odot 2003$ Society for Neuroscience $\quad$ 0270-6474/03/2311628-13\$15.00/0 tical cells to injection of a broadband noisy input is linear and can be flat up to $100 \mathrm{~Hz}$. Bair and Koch (1996), using in vivo recordings in middle temporal (MT) cortex of anesthetized monkeys, showed that the power spectra of the responses of neurons to randomly moving dots are low-pass with a broad range of cutoffs up to $150 \mathrm{~Hz}$.

The linear response of neurons to noisy fluctuating inputs has also been investigated in theoretical studies relying on the leaky integrate-and-fire (LIF) model (Knight, 1972a; Gerstner, 2000; Brunel et al., 2001; Fourcaud and Brunel, 2002; Mazurek and Shadlen, 2002). It was found that in the presence of white noise, LIF neurons behave like low-pass filters, with a cutoff depending on the passive membrane time constant and the average firing rate of the neuron. The gain of the filter decays as $1 / \sqrt{f}$, where $f$ is the input frequency, and its phase shift reaches the value of $45^{\circ}$, at sufficiently large $f$. It was also found that temporal correlations in the input noise improve the "accuracy" of the LIF response, because they suppress the decay at high frequencies and reduce the phase shift (Gerstner, 2000; Brunel et al., 2001; Fourcaud and Brunel, 2002). In the present study, we show that simple conductance-based (CB) models behave differently. Using a combined analytical and numerical approach, we investigated theoretically how neuronal properties affect the response of neurons to fluctuating inputs. In particular, we demonstrate that the suppression of the AC part of the spike response to fast varying inputs depends on the DC response of the neuron and on the characteristics of the fast sodium currents responsible for spike initiation. 


\section{Materials and Methods}

Linear response of the instantaneous firing rate. We investigated the response of a neuron to a time-varying input. Our goal is to determine the instantaneous firing rate $\nu(t)$ (i.e., the probability that the neuron will fire between time $t$ and time $t+d t$, divided by $d t$, in the limit $d t \rightarrow 0$ ). We assume that the neuron receives an input

$$
I_{\text {syn }}(t)=I_{0}+\delta I(t)+I_{\text {noise }},
$$

where $I_{0}$ is the DC component of the input, $\delta I(t)$ is its deterministic temporal variation around $I_{0}$, and $I_{\text {noise }}$ is a noisy component that is described in more detail below. The firing rate $\nu(t)$ is determined as a function of $I_{0}+\delta I(t)$ by averaging the response of the neuron over different realizations of $I_{\text {noise }}$. Equivalently, $\nu(t)$ represents the instantaneous firing rate of a population of noninteracting neurons that receive the same input $I_{0}+\delta I(t)$, but in which each neuron receives an independent realization of the noise.

In the limit of small $\delta I(t)$, a linear relationship can be written between $\nu(t)$ and $\delta I(t)$ :

$$
\nu(t)=\Phi\left(I_{0}\right)+\int_{0}^{\infty} d t^{\prime} \kappa\left(t^{\prime}\right) \delta I\left(t-t^{\prime}\right),
$$

where $\Phi\left(I_{0}\right)$ is the average firing rate of the neuron in response to a current $I_{0}$ in the presence of noise $I_{\text {noise }}$ (the $f-I$ curve of the neuron), and the kernel, $\kappa$, depends on $I_{0}$ and the properties of the noise. In particular, the response to a sinusoidally and weakly modulated $I_{\text {syn }}(t)$ :

$$
I_{\text {syn }}(t)=I_{0}+I_{1} \cos (2 \pi f t)+I_{\text {noise }},
$$

where $I_{1} \ll I_{0}$ is:

$$
\nu(t)=\nu_{0}+\nu_{1}(f) \cos [2 \pi f t+\phi(f)],
$$

where $\nu_{0}=\Phi\left(I_{0}\right)$, and $\nu_{1}(f)$ and $\phi(f)$ are related to the modulus and the argument of the Fourier transform of $\kappa, \hat{\kappa}(f)$, by $\nu_{1}(f)=I_{1}|\hat{\kappa}(f)|$ and $\phi(f)=\arg \hat{\kappa}(f)$.

The gain of the response at frequency $f, \nu_{1}(f) / I_{1}$, and the phase response, $\phi(f)$, completely characterize the linear filtering properties of the neuron. We compute these quantities as a function of the frequency $f$. Note that at sufficiently low frequencies, it is straightforward to relate the gain $\nu_{L F} / I_{1}$ to the $f-I$ curve of the neuron. Indeed, for small $f$, one can write $\nu(t)=\Phi\left[I_{0}+I_{1} \cos (2 \pi f t)\right]$, so that because $I_{1}<<I_{0}$ :

$$
\frac{\nu_{L F}}{I_{1}}=\frac{\partial \Phi}{\partial I},
$$

whereas the phase shift at small $f$ is $\phi_{L F}=0$.

Noise. When a neuron receives a large number of synaptic inputs per membrane time constant through synapses with small amplitude compared with threshold, the resulting currents can be well approximated by a mean input current with random Gaussian variations around the mean (diffusion approximation) (Tuckwell, 1988); thus, we consider Gaussian noise. If one assumes that postsynaptic currents are instantaneous, the Gaussian noise is white. For exponentially decaying synaptic currents, the Gaussian noise is colored with a correlation time equal to the synaptic decay time constant (see Appendix B2).

\section{Conductance-based models}

Two single-compartment neuronal models are used in this study. The first was proposed by Wang and Buzsáki (1996), which is a modified version of the original Hodgkin-Huxley model (Hodgkin and Huxley, 1952). The Wang-Buzsáki (WB) model has a leak current, a fast sodium current with instantaneous activation dynamics, $m(V)$, and a delayedrectifier potassium current. The firing current threshold of the neuron is $I_{T}=0.16 \mu \mathrm{A} / \mathrm{cm}^{2}$. This corresponds to a voltage threshold $V_{T}=-59.9$ $\mathrm{mV}$. Note that the WB model is type I (zero firing frequency at threshold) and not type II (nonzero firing frequency at threshold), like the Hodgkin-Huxley model with standard parameters (Hodgkin and Hux- ley, 1952) because of the different choice of parameters, in particular for the sodium and potassium conductance (Wang and Buzsáki, 1996).

The second model was proposed by Hansel and van Vreeswijk (2002), which has the fast sodium current and the delayed rectifier current of the WB model but also additional intrinsic currents: A-type current, slow potassium current, and persistent sodium current. Details of the models are given in Appendix A.

Numerical integration of $C B$ models. Model equations in the presence of noise have been integrated using a stochastic second-order Runge Kutta (RK2) method (Honeycutt, 1992), with a time step $<0.02 \mathrm{msec}$ in all simulations. The instantaneous firing rate was computed by taking the average spike count in bins of one-thirtieth of the input period over a minimum of 2000 periods. The obtained instantaneous firing rate was fitted by a sinusoid (Eq. 4) using a least-square method or by computing directly the first Fourier component of the output spike train. Both methods yielded the same results. The input modulation amplitude $I_{1}$ was adjusted depending on the input frequency to get an instantaneous firing rate modulation equal to $25 \%$ of the baseline. We checked that, to this level of input modulation, the amplitude of the firing rate modulation $\nu_{1}$ varied linearly with $I_{1}$.

\section{Nonlinear integrate-and-fire neuron models}

To understand the factors that determine the response to time-varying inputs, we investigated simpler models that are to some extent analytically tractable. We introduced a family of one-variable neuronal models whose membrane potential $V$ dynamics are given by:

$$
C \frac{d V}{d t}=-g_{L}\left(V-V_{L}\right)+\psi(V)+I_{\text {syn }}(t),
$$

where $C$ is the membrane capacitance, $g_{L}$ is the leak conductance, $V_{L}$ is the leak potential, $I_{\text {syn }}$ is the external synaptic current (Eq. 3 ), and $\psi(V)$ is a function of voltage that describes the spike-generating currents. The passive membrane time constant is $\tau_{m}=C / g_{L}$.

The LIF (Knight, 1972a; Tuckwell, 1988) is the special case of this family of models for which $\psi(V)=0$. In this model, a spike threshold $V_{\text {th }}$ has to be imposed to obtain spike generation. The resulting spike is instantaneous, and the neuron is reset to a voltage $V_{r}$ after a spike.

When the function $\psi(V)$ is supralinear, the membrane potential diverges to infinity in finite time if the input current exceeds some threshold. This divergence can be identified with the firing of a spike, provided that one supplements Equation 6 with a reset condition for the membrane potential to a value $V_{r}$, as in the LIF model. A threshold voltage $V_{T}$ can be defined as the voltage at which the slope of the $I-V$ curve vanishes, which is given by:

$$
\psi^{\prime}\left(V_{T}\right)=g_{L}
$$

This is also the largest steady voltage at which the neuron can be maintained by a constant input current. The corresponding current $I_{T}=$ $g_{L}\left(V_{T}-V_{L}\right)-\psi\left(V_{T}\right)$ is the threshold current above which tonic firing occurs. We also defined the spike slope factor $\Delta_{T}$ (in $\mathrm{mV}$ ) as:

$$
\Delta_{T}=\frac{g_{L}}{\psi^{\prime \prime}\left(V_{T}\right)} .
$$

This parameter, which is inversely proportional to the curvature of the $I-V$ curve at the threshold $V_{T}$, measures the sharpness of spike initiation. The quadratic integrate-and-fire (QIF) neuron corresponds to:

$$
\psi(V)=\frac{g_{L}}{2 \Delta_{T}}\left(V-V_{T}\right)^{2}+g_{L}\left(V-V_{L}\right)-I_{T}
$$

where $I_{T}$ is the current threshold, $V_{T}$ and $\Delta_{T}$ are the spike threshold and slope factor, respectively, as defined above. If one chooses $V_{r}=-\infty$, this model represents the normal form of type I neurons (Ermentrout and Kopell, 1986; Ermentrout, 1996).

Other spike-generating currents, $\psi(V)$, were considered in this study. 
Of particular interest is the exponential integrate-and-fire (EIF) neuron for which the spike-generating current is exponential:

$$
\psi(V)=g_{L} \Delta_{T} \exp \left(\frac{V-V_{T}}{\Delta_{T}}\right)
$$

It is easy to check that $V_{T}$ and $\Delta_{T}$ satisfy Equations 7 and 8, respectively. Note that in the limit $\Delta_{T} \rightarrow 0$ (spike with very sharp initiation), the EIF neuron becomes equivalent to the LIF model with $V_{\text {th }}=V_{T}$.

Fitting IF to the Wang-Buszáki model. In all of the models considered in this study, we take $C=1 \mu \mathrm{F} / \mathrm{cm}^{2}, g_{L}=0.1 \mathrm{mS} / \mathrm{cm}^{2}$, and $V_{L}=-65 \mathrm{mV}$. The spike threshold $V_{\text {th }}$ of the LIF model was chosen such that the threshold current is equal to the threshold current $I_{T}$ of the WB model. In the WB model, the $f-I$ curve behaves near the onset of firing $\left(I \gtrsim I_{T}\right)$ as $\beta \sqrt{\mathrm{I}-\mathrm{I}_{\mathrm{T}}}$. This is also the case for the QIF and EIF models. Therefore, one can determine the parameters $\beta$ and $I_{T}$ to match the behavior of their $f-I$ curves with one of the WB models. This gives $\beta=$ $0.038 \mathrm{msec}^{-1} \mu \mathrm{A}^{-1 / 2} \mathrm{~cm}, I_{T}=0.16 \mu \mathrm{A} / \mathrm{cm}^{2}$. This also determines $\Delta_{T}=$ $3.48 \mathrm{mV}$ and $V_{T}=-59.9 \mathrm{mV}$. For larger $I$, the $f--I$ curves are no longer well described by the square root behavior. The reset voltage, $V_{r}$, and the refractory period, $\tau_{\text {ref }}$, were chosen to minimize the difference of its $f-I$ curve with one of the WB models for input currents well above $I_{T}$ (Fig. 1). Matching the $f-I$ curves in this range of currents determines $V_{r}=-63.8$ $\mathrm{mV}$ and $\tau_{\text {ref }}=0$ (QIF model), $V_{r}=-68 \mathrm{mV}$ and $\tau_{\text {ref }}=1.7 \mathrm{msec}$ (EIF model).

Fokker-Planck equation. To analyze the response of an IF neuron to a time-dependent input, we studied how the distribution of its voltage, $P(V, t)$, evolves over time. We assumed Gaussian white noise, $I_{\text {noise }}(t)=\sigma \sqrt{C_{g_{L}}} \eta(\mathrm{t})$, where $\eta(t)$ has unit variance and $\sigma$ has a dimension of a voltage. The distribution, $P(V, t)$, at time $t$ obeys the FokkerPlanck equation (Risken, 1984; Abbott and van Vreeswijk, 1993; Brunel and Hakim 1999; Brunel, 2000; Knight et al., 2000; Nykamp and Tranchina, 2000; Brunel et al., 2001; Fourcaud and Brunel, 2002):

$$
\frac{\partial P}{\partial t}=-\frac{\partial J_{V}}{\partial V}
$$

where

$$
J_{v}(V, t)=\left[-\frac{\left(V-V_{L}\right)}{\tau_{m}}+\frac{\psi(V)+I(t)}{C}\right] P(V, t)-\frac{\sigma^{2}}{2 \tau_{m}} \frac{\partial P}{\partial V},
$$

is the probability flux through the potential $V$ (i.e., the probability per unit of time that the membrane potential of the neuron crosses the value $V$ from below at time $t$ ). The firing rate, $\nu$, of the neuron is the flux through $V=\infty$ :

$$
\nu(t)=\lim _{V \rightarrow+\infty} J_{V}(V, t) .
$$

For a complete analytical description, one also needs the boundary conditions, which take into account that, after the spike, the neuron is reset to $V_{r}$.

All of our calculations are based on the above equations, which are valid for white input noise, or on their generalizations for more realistic noise models. The details of the calculations can be found in Appendix B.

Simulations of the EIF model. In the EIF model, an action potential is defined as a divergence of the voltage. In simulations, one has to introduce a cutoff at a finite voltage $V_{\text {th }}$. However, if one assumes that the neuron immediately spikes after reaching $V_{\text {th }}$, one ends up with a neuron that is effectively an LIF for some inputs. This can be avoided by treating the spike more carefully. In our simulations, we chose a threshold $V_{\text {th }}$ large enough so that:

$$
\psi\left(V_{\mathrm{th}}\right) \gg I_{0}+I_{1}+I_{L}+\sigma \sqrt{C g_{L}} .
$$

by a factor $\geq 100$. For $V<V_{\mathrm{th}}$, we integrated the dynamics using a stochastic RK2 algorithm (Honeycutt, 1992). For $V>V_{\text {th }}$, we neglected all of the currents but $\psi(V)$ and analytically integrated Equation 6 . In particular, the time it takes for the voltage to increase from $V=V_{\text {th }}$ to
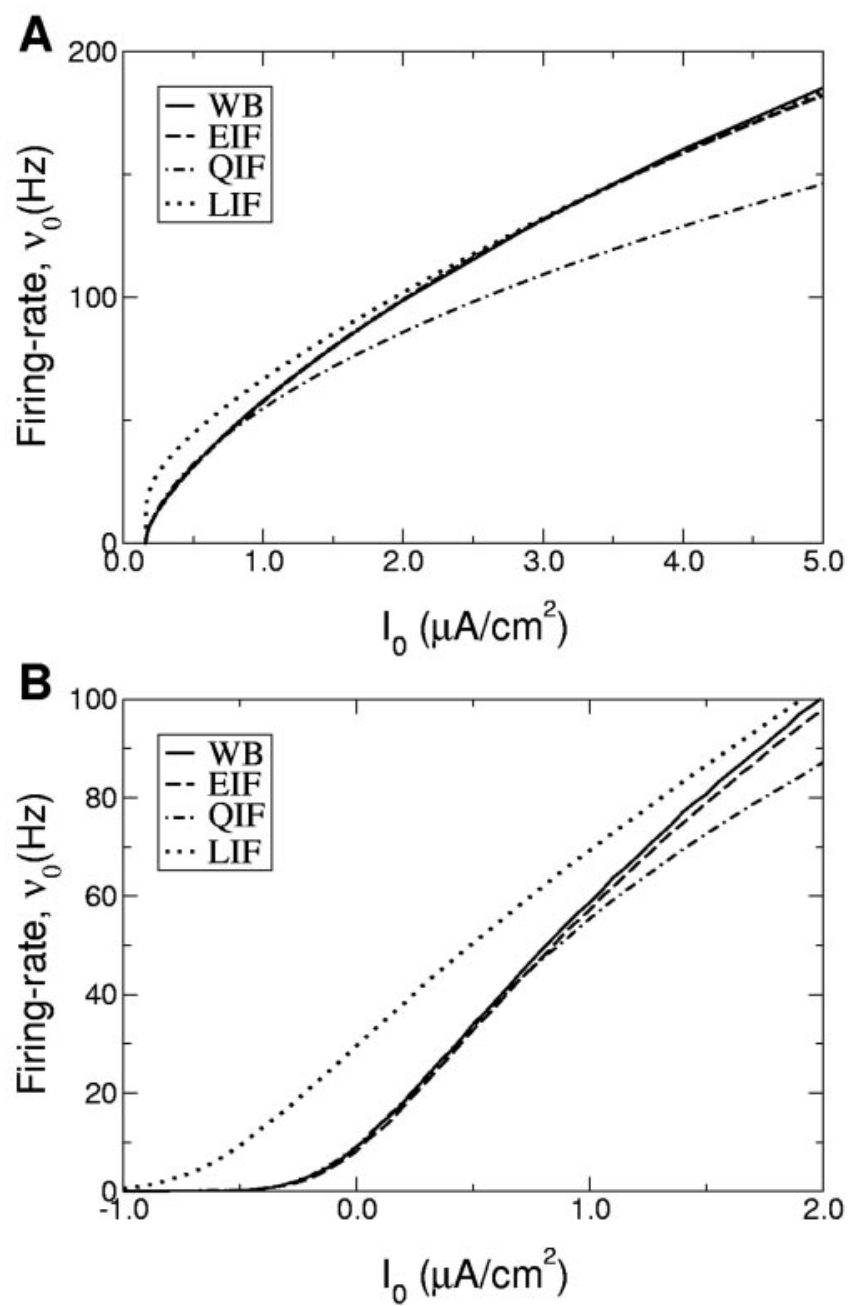

Figure 1. $\quad A, B, f-I$ curves of the LIF, QIF, EIF, and WB neurons for a constant input current $(A)$ and a noisy input current $(B)$ (Gaussian white noise, $\sigma=5 \mathrm{mV}$ ). The parameters of the EIF model were chosen to match the $f-I$ curve of the WB model. The parameters of the QIF model were chosen to match the behavior of the $f-I$ curve of the WB model near firing onset. The range of firing rates in which the $f-I$ curves of the QIF and WB models match is more restricted than for the EIF model. The $f-I$ curve of the LIF neuron cannot be made to agree with the $f-I$ curves of the other models at low firing rates because of the different qualitative dependence of the firing rate on the input current (logarithmic vs square-root). In contrast, the parameters of the LIF model can be determined to match the $f-I$ curve of the WB model at high frequencies (see Materials and Methods for details on the determination of the model parameters).

$V=+\infty$ is $t_{\mathrm{sp}}=\tau_{m} \exp \left[\left(V_{T}-V_{\mathrm{th}}\right) / \Delta_{T}\right]$. If $V_{\text {th }}$ is too large, the RK2 procedure at large $V$ underestimates the increase in the membrane potential at each time step. This leads to a systematic overestimate in the spike time and causes an additional phase shift in the neuronal response. In practice, we must make sure the time $t_{\mathrm{sp}}$ is large compared with the integration time step $d t$. Thus, one needs to check that the two conditions, (Eq. 13) and $t_{\mathrm{sp}} \gg d t$, are both satisfied. In the simulations with $\Delta_{T}=3.48$, a value for which both conditions are satisfied with $V_{\mathrm{th}}=-30$ $\mathrm{mV}$ for the range of inputs that we investigated.

\section{Results}

\section{Filter of a simple conductance-based model}

We investigated how the instantaneous firing rate of the $\mathrm{WB}$ model is modulated by a sinusoidal input at frequency $f$ in the presence of a noisy background input using numerical simulations. Figure $2 \mathrm{~A}$ shows a particular realization of such an input current. The instantaneous firing rate is computed by averaging the response over many realizations of the noise, as shown in 

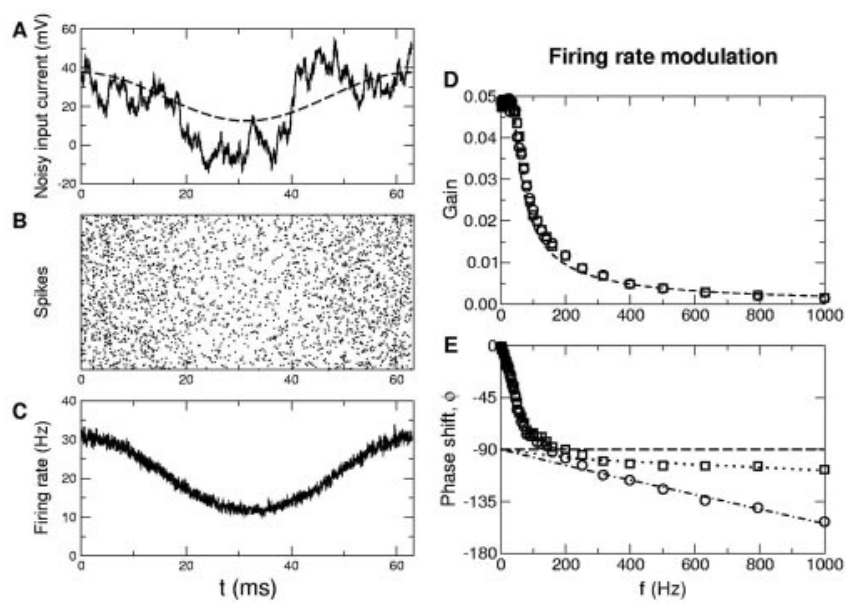

Figure 2. Firing rate modulation of a conductance-based neuron. $A$, Deterministic part of the input current (dashed line) with input noise (solid line). $B$, Raster plot, 2000 repetitions of the input current with independent noise sources. $C$, Instantaneous firing rate of the neuron averaged over these repetitions. $D, E$, Gain $(D)$ and phase shift $(E)$ of the firing rate modulation of a WB neuron versus the frequency, $f$, of the oscillating input. Spiking threshold, $V_{s}$ is $-20 \mathrm{mV}$ $(\square)$ and $20 \mathrm{mV}(\bigcirc)$. The dashed line in $D$ is obtained by fitting the simulated data, assuming a decay proportional to $1 / f$ at high frequencies. The phase shift at high frequencies depends on the definition of the spike time. It increases linearly with $f$ (dotted and dotted- dashed lines are linear fits at high frequencies). In both $D$ and $E$, error bars are smaller than symbol size. Parameters: $\sigma=6.3 \mathrm{mV}$ (white noise), $\nu_{0}=40 \mathrm{~Hz}$.

Figure 2, $B$ and $C$. Figure 2, $D$ and $E$, shows how the amplitude and phase shift of the firing rate modulation of the neuron depends on the frequency of the sinusoidal input when the noisy component is a Gaussian white noise with an SD of $\sigma=6.3 \mathrm{mV}$ and its DC part is such that the average firing rate is $\nu_{0}=40 \mathrm{~Hz}$.

The amplitude versus frequency curve (Fig. 2D) shows a marked attenuation of the response above a frequency of $\sim 50 \mathrm{~Hz}$. A fit of our numerical simulation data in the range $50 \mathrm{~Hz}<f<$ $500 \mathrm{~Hz}$, assuming a power law decay, reveals that the firing rate modulation at large $f$ decays as $1 / f$ (Fig. $2 D$ ). Moreover, we find that in this frequency range, the phase shift does not vary much and is slightly larger than $90^{\circ}$, as shown in Figure $2 \mathrm{E}$. We performed extensive numerical simulations varying the level of noise and the DC external input as well as the temporally correlated noise. In all of these simulations, the same behavior of the firing rate modulation was found at sufficiently large $f$, although the frequency above which it becomes apparent depends on the values of the varied parameters (Fig. 9).

In all of these simulations, the instantaneous spike rate was computed using a definition of spike time as the time the membrane potential crosses a voltage, $V_{s}=20 \mathrm{mV}$, from below. The response modulation amplitude does not depend on the precise value of $V_{s}$, provided it is sufficiently high (more than $-30 \mathrm{mV}$ ). In contrast, the phase shift is more sensitive to $V_{s}$, especially at very high input frequencies where the increase of the phase shift is linear with a slope that depends on $V_{s}$ (Fig. $2 E$ ).

\section{Integrate-and-fire models with intrinsic spike generation mechanisms}

Recent studies have shown that the high-frequency response of the LIF model depends on the temporal correlations of the noise. For white noise, the response decreases as $1 / \sqrt{f}$ with a phase lag of $45^{\circ}$; for colored noise, the response stays finite with no phase lag (Brunel et al., 2001; Fourcaud and Brunel, 2002). These results are clearly at odds with what we found in the simulations of the WB model.
To understand the origin of the behavior of the WB model at high frequencies and why it is different from the LIF, we studied nonlinear integrate-and-fire neurons, in which the membrane potential obeys Equation 6. The advantage of using this simplified model is that it allows us to compute analytically both the $f-I$ curve and the firing rate modulation at high frequencies, and hence to understand in detail the factors that affect it.

With a supralinear spike-generating current $\psi(V)$, the neuron is endowed with an intrinsic mechanism for spike initiation. We considered two cases in particular, the QIF neuron defined by Equation 9 and the EIF neuron defined by Equation 10. These two neuronal models are type I, like the WB model, and their $f-I$ curve increases proportionally to $\sqrt{I-I_{\mathrm{T}}}$, near the firing onset at current threshold, $I_{T}$. Hence, for both the QIF and EIF, the parameters can be chosen so that the onset of firing in response to a constant input reproduces the parameters of the WB model sufficiently close to the current threshold (Fig. 1) (see Materials and Methods for details). For the EIF model, this constraint determines the value of the "spike slope factor," $\Delta_{T}$. To determine the remaining free parameters, $V_{r}$ and $\tau_{\text {ref, }}$, we also required a good fitting of the WB model $f-I$ curve outside the region of bifurcation.

The $f-I$ curves of the QIF and EIF, with parameters so defined, and the WB model are compared in Figure $1 A$. It shows that the firing rate response to constant current of the EIF and WB models are very close in a broad range of firing rates (up to $200 \mathrm{~Hz}$ ). The QIF neuron reproduces the $f-I$ curve of the WB neuron well at low firing rates but not at high rates. Finally, the LIF neuron cannot reproduce the $f-I$ curve of type I neurons well at low rates, because the firing rate has a logarithmic, not square root, dependence on $I-I_{T}$. However, the LIF neuron can be made to reproduce the $f-I$ curve reasonably well at high firing rates by a suitable adjustment of parameters.

We then compared the response of the QIF, EIF, LIF, and WB models to noisy input currents (Eq. 3, with $I_{1}=0$ ). The corresponding $f-I$ curves are plotted in Figure $1 B$ for the four models with the same parameters as in Figure $1 A$. As in the noiseless case, the $f-I$ curves of the QIF and EIF models match the WB model $f-I$ curve very well in a broad range of input currents, and this range is larger for the EIF model than for the QIF model.

For a more in-depth look at the spiking dynamics of these various models, Figure 3 plots the voltage traces of the four models for the same realization of the input current. As shown in Figure $3 A$, the QIF, EIF, and LIF models behave on large time scales in a very similar way as the WB model. However, differences between these models are found on shorter time scales, as shown in Figure $3 B$. The best match with the WB model is obtained with the EIF model. The subthreshold potential traces are essentially indistinguishable. This is because the same leak current is present in both models and because the $I-V$ curves of the two models are very similar near threshold voltage, $V_{T}$. The latter property stems from the fact that the activation curve of the fast sodium current in the WB model can be well approximated near threshold by an exponential function, as shown in Figure 3C. Thus, the membrane potential behavior at the spike onset is very similar for the WB and EIF models. After initiation, the dynamics of the spike in the WB model is controlled by the interplay between $\mathrm{Na}^{+}$and $\mathrm{K}^{+}$channel dynamics and is essentially independent of synaptic inputs. Thus, its spike shape is approximately invariant. Consequently, the only significant difference between the EIF and WB models, the precise shape of the spike after the 
A

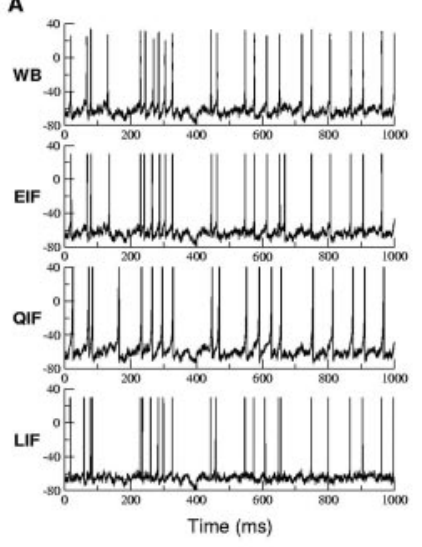

B

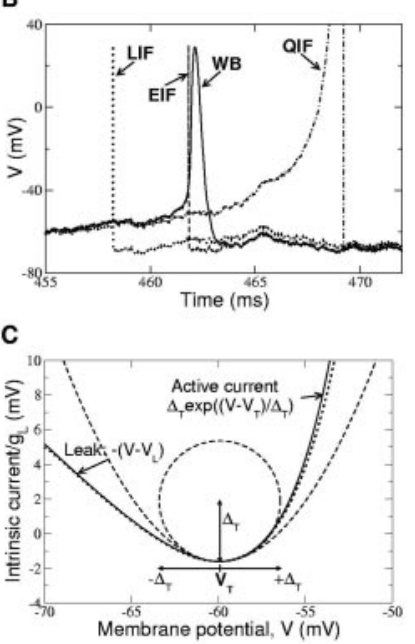

Figure 3. A, Voltage traces for WB, EIF, QIF, and LIF neurons for the same realization of the noisy input current. $B$ shows a higher resolution for a short time interval in which a spike has been generated in all models. The subthreshold traces are similar for all models; however, the dynamics of the spike are different on an msec time scale. When the fluctuation leads to a spike in all models, the LIF neuron spikes first. The EIF neuron spikes almost exactly at the spike onset of the WB. The QIF neuron fires much later. For details of the QIF and EIF parameters, see Materials and Methods. For the WB parameters, see Appendix A. Here, the LIF model has the leak current of the WB model, a reset potential of $V_{r}=-68 \mathrm{mV}$, and $V_{\text {th }}=-57 \mathrm{mV}$, to get the same average firing rate as the WB model. C, I-V curve of the EIF (solid line) and WB (dotted line) neurons. The threshold $V_{T}$ is defined as the minimum of the curve. The spike slope factor $\Delta_{T}$ is proportional to the radius of the curvature of the $I-V$ curve at its minimum.

onset, leads to an input-independent fixed short delay (of order $0.1-0.2 \mathrm{msec}$ ) between the spikes of the two models.

The other two models failed to reproduce the dynamics of spike initiation of the WB model. When a fluctuation in the input current induces a spike in all four models, the LIF neuron, which has an instantaneous spike, usually spikes first. The spike in the QIF neuron usually occurs later because of the long time spent by the neuron in the voltage threshold.

\section{The high-frequency response of nonlinear integrate-and-fire neurons}

We analytically calculated the firing rate response of a general IF model to a sinusoidally modulated input in the presence of Gaussian white noise (Eq. 3). The details of the calculations, which rely on the Fokker-Planck equation and an expansion of both instantaneous firing rate and membrane potential distribution in powers of $1 / f$, are described in Appendix B. There, we prove that, depending on the function $\psi(V)$, there are three classes of possible behaviors for the firing rate modulation at high frequencies:

Table 1. High-frequency properties of linear and nonlinear integrate-and-fire models

\begin{tabular}{llc}
\hline Model & Exponent & Phase lag \\
& $\alpha$ & $\phi(f \rightarrow \infty)$ \\
\hline LIF, colored noise & 0 & 0 \\
LIF, white noise & 0.5 & $45^{\circ}$ \\
EIF, all types of noise & 1 & $90^{\circ}$ \\
QIF, all types of noise & 2 & $180^{\circ}$ \\
\hline
\end{tabular}

The amplitude of response modulation in the high-frequency regime is proportional to $1 / f^{\alpha}$. Note that the types of noise considered include current-based as well as conductance-based synaptic inputs.

(1) If $\psi^{\prime}(V) / \psi(V)$ does not vanish and is finite at large $V$, the phase lag $\phi(f)$ is $90^{\circ}$, whereas the response modulation is given by:

$$
\nu_{1}(f)=\frac{I_{1} \nu_{0}}{2 \pi C f} \lim _{V \rightarrow \infty} \frac{\psi^{\prime}(V)}{\psi(V)},
$$

where $\psi^{\prime}(V)$ is the derivative of $\psi$. This happens if the spikegenerating current has an exponential dependence on the voltage, as in the EIF model. For this model, one finds that at high frequencies:

$$
\nu_{1}(f)=\frac{\nu_{0} I_{1}}{2 \pi C \Delta_{T} f},
$$

and the phase lag is $\phi=90^{\circ}$.

(2) If $\psi^{\prime}(V) / \psi(V)$ goes to zero at large $V$, the rate modulation decays faster than $1 / f$ at high frequencies. This is, for instance, the case of the QIF model for which the calculation of the order $1 / f^{2}$ in the expansion of $\nu_{1}$ shows that at high frequencies, $\nu_{1}(f) \approx$ $\left(\nu_{0} I_{1}\right) /\left[g_{L} \Delta T\left(2 \pi f \tau_{m}\right)^{2}\right]$, and the phase lag is $\phi=180^{\circ}$ (see Appen$\operatorname{dix} B)$.

(3) If $\psi^{\prime}(V) / \psi(V)$ diverges when $V$ goes to infinity, the response is less attenuated than $1 / f$. This happens in particular for the LIF neuron.

We also show in Appendix B that in cases 1 and 2, the highinput frequency behavior is independent of the temporal correlations of the noise. In contrast, in case 3, the properties of the noise matter, as found in previous work in which the LIF model was studied (Brunel et al., 2001; Fourcaud and Brunel 2002). Furthermore, it can be shown that the results hold for currentbased as well as conductance-based noise, because in the case of suprathreshold membrane potential, the spike-generating current $\psi(V)$ dominates the neuronal dynamics, and the synaptic input has little effect on what happens after spike initiation.

In conclusion, the amplitude of the firing rate modulation behaves at high frequencies like a power law $\nu_{1}(f) \approx 1 / f^{\alpha}$, where the exponent $\alpha$ depends on the nonlinearity of the spikegenerating current $\psi(V)$. The phase lag at high-input frequency is $\alpha \pi / 2$. The exponent $\alpha$ and asymptotic phase shift of the various models are summarized in Table 1 . The high frequencies behaviors of the rate modulation in WB and EIF models are extremely consistent. Other IF models failed to reproduce the behavior of the WB model.

\section{Properties of the EIF neuron at intermediate frequencies}

The simulation and analytical results described so far show that in the limit of low- and high-input oscillation frequencies, the behaviors of the EIF and WB models are very similar. We consider now the behavior of the EIF model at intermediate frequencies using numerical simulations.

When the noise level is weak, resonances appear at frequencies that are integer multiples of the average firing rate $\nu_{0}, f=k \nu_{0}(k=$ $1,2, \ldots$ ) (Fig. 4, insets). This is because at low noise levels, the neuron behaves like an oscillator. When the noise increases, the neuronal firing process becomes less regular. The resonant peaks become less pronounced and they disappear, one after the other. For a sufficiently high noise level, the last resonance peak $(k=1)$ disappears, and the firing rate modulation monotonically decreases with the input frequency, $f$. The dashed line in Figure 4, computed by numerical simulations, represents the boundary in the plane $\left(\nu_{0}, \sigma\right)$ between the weak noise regime (below the line) where the firing rate modulation displays resonances and the 


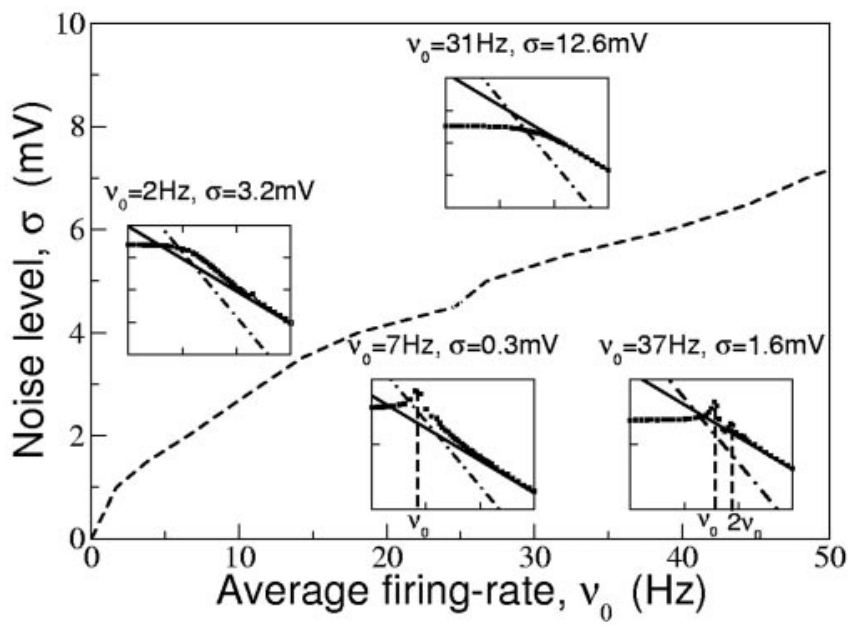

Figure 4. The qualitative behaviors of the firing rate modulation of the EIF model at intermediate frequencies depend on the characteristics of the input current. Insets show a representative example of the corresponding behavior (gain vs input frequency in log-log scale). In all insets, we plot a simulation of the EIF model (squares, gain) and the EIF and QIF high input frequency regimes (solid and dotted-dashed lines). The dashed line gives the noise level below which there are resonant peaks at the average firing rate $\nu_{0}$ and possibly at integer multiples of $\nu_{0}$ in response. Above the dashed line, the EIF model behaves like a low-pass filter, with approximately constant gain at low frequencies and a $1 / f$ attenuation for sufficiently high frequencies. The gain reaches the asymptotic behavior from above at low firing rates, whereas it reaches the asymptotic behavior from below for high firing rates and high noise (see differences in the two insets in the high noise region).

strong noise regime (above the line) where the resonances are completely suppressed. In the latter region, the spike response modulation, $\nu_{1}(f)$, is approximately constant in some range of input frequencies, beyond which it decreases with $f$ with an asymptotic decay in $1 / f$. Therefore, when the noise is sufficiently large, the EIF neuron behaves like a low-pass filter.

The gain and phase shifts of the filter in the presence of white noise are plotted in Figure 5, $A$ and $B$, respectively, for fixed $\nu_{0}$ and three values of the spike slope factor, $\Delta_{T}$. In all cases, the gain of the filter decreases as $1 / f$ at large enough frequencies. This asymptotic regime is reached earlier for larger $\Delta_{T}$. There is an overall reduction of the gain as $\Delta_{T}$ increases. This effect is in agreement with our analytical calculation (Eq. 15), which predicts that in the limit $f \rightarrow \infty$, the firing rate modulation, $\nu_{1}$, decreases with $\Delta_{T}$. This is also consistent with Equation 5 , because one can show (using Eq. 29) that the derivative of the $f-I$ curve at $\nu_{0}=20 \mathrm{~Hz}$ is a decreasing function of $\Delta_{T}$. The phase shift is an increasing function of $\Delta_{T}$, as shown in Figure $5 B$. This corresponds to what is expected intuitively, because a decrease of $\Delta_{T}$ increases the sharpness of spike initiation. At large frequencies, the phase shift of the filter goes to $-\pi / 2$ for any $\Delta_{T}$, but the convergence is slower when $\Delta_{T}$ is small.

Figure 6 shows the effect of temporal correlations in the input noise on the shape of the filter. The response is plotted for three values of the temporal correlation of the noise, $\tau_{s}$, over a broad range of input frequencies, $f$. In these simulations, the noise level was adjusted so that the low-frequency response stays constant as $\tau_{s}$ was varied. All three curves overlap for sufficiently large frequencies, $f>100 \mathrm{~Hz}$, in agreement with our analytical results. In contrast, for intermediate frequencies, $30<f<100 \mathrm{~Hz}$, the response modulation increases with $\tau_{s}$. This effect is reminiscent of the behavior of the LIF neuron (Brunel et al., 2001) for which correlations in the input noise suppresses the attenuation of the response modulation (Brunel et al., 2001; Fourcaud and Brunel, 2002).
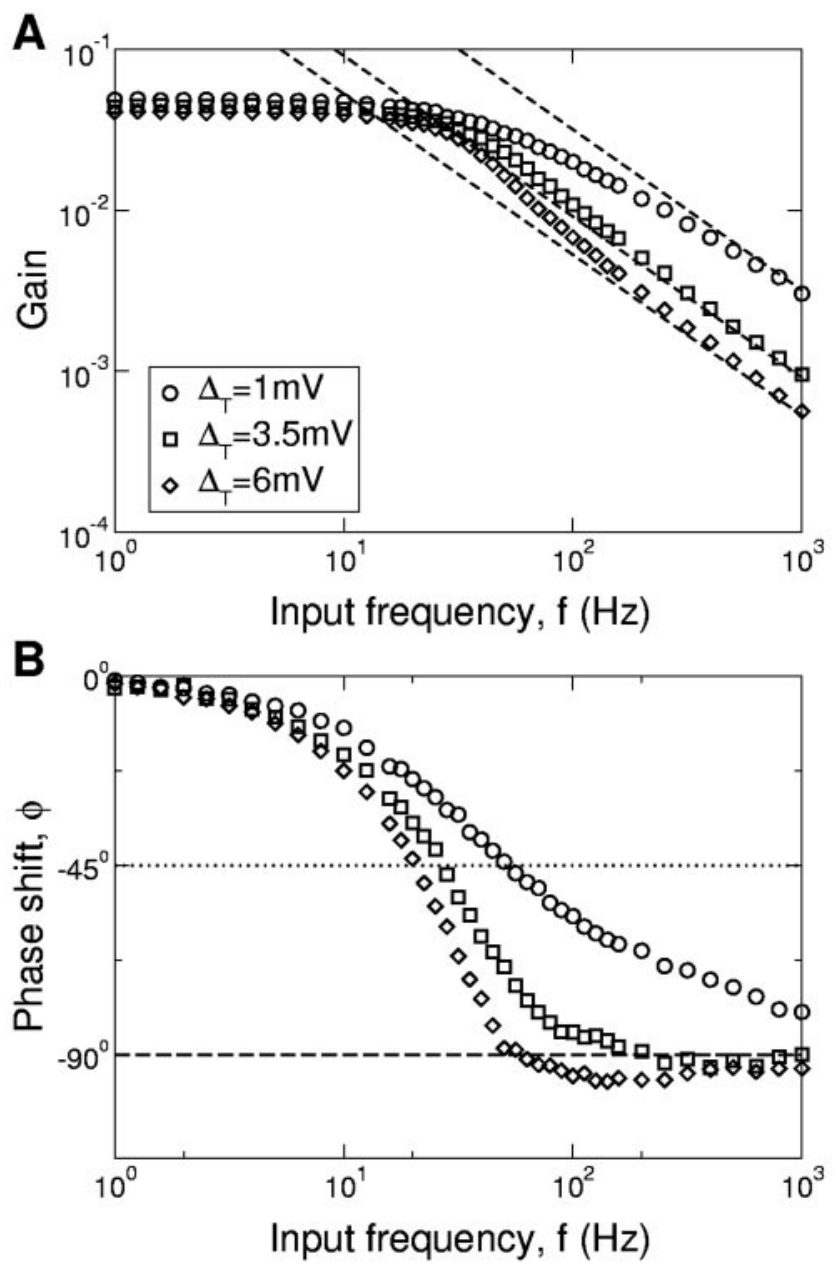

Figure 5. Influence of the spike sharpness on the EIF filter. $A, B$, Gain $(A)$ and phase $(B)$ of the firing rate modulation are plotted for different values of the spike slope factor $\Delta_{T}$, indicated in A. Other parameters: $\nu_{0}=20 \mathrm{~Hz}, \sigma=6.3 \mathrm{mV}$. Note that as $\Delta_{T}$ decreases, the high frequency asymptotic regime is shifted to higher input frequencies. For large $\Delta_{T}$, the gain decays faster than $1 / f$, and the phase shift is larger than $90^{\circ}$ in an intermediate frequency range.

To further characterize the filtering properties of the neuron, we defined the filter cutoff as the frequency, $\mathrm{f}_{c}$, for which the filter gain decreases by a factor $1 / \sqrt{2}$ compared with its low-frequency limit, $\nu_{1}\left(f_{c}\right)=\nu_{L F} / \sqrt{2}$. We used numerical simulations to study how $f_{c}$ depends on the average firing rate, $\nu_{0}$, the spike slope factor, $\Delta_{\mathrm{T}}$, and the temporal correlations in the noise. Figure 7 shows the results for a noise level $\sigma=8 \mathrm{mV}$. For this value of $\sigma$, the EIF neuron is in the strong noise regime in the entire range of average firing rates and the spike slope factor explored.

Figure $7 A$ shows that for a fixed value of the slope factor, $f_{c}$ is finite in the limit of low average firing rate, $\nu_{0}$, and that it is an increasing function of $\nu_{0}$. For $\nu_{0}>10 \mathrm{~Hz}, f_{c}$ varies linearly with $\nu_{0}$. In Figure $7 B$, the cutoff frequency is displayed as a function of $\Delta_{T}$ for fixed $\nu_{0}$ and different values of $\tau_{s}$, the noise correlation time. In general, $f_{c}$ is a decreasing function of $\Delta_{T}$ but, at large enough $\Delta_{T}$, it saturates toward a value that depends only weakly on $\tau_{s}$. In contrast, the smaller the $\tau_{s}$, the weaker the variations of $f_{c}$ at small $\Delta_{T}$. In particular, for white input noise, $f_{c}$ is almost constant in all of the explored range of $\Delta_{T}$. Temporal correlations in the noise increase the cutoff frequency, $\mathrm{f}_{c}$, and this effect is more pronounced at smaller values of $\Delta_{T}$ (i.e., when spikes have sharper initiation). 


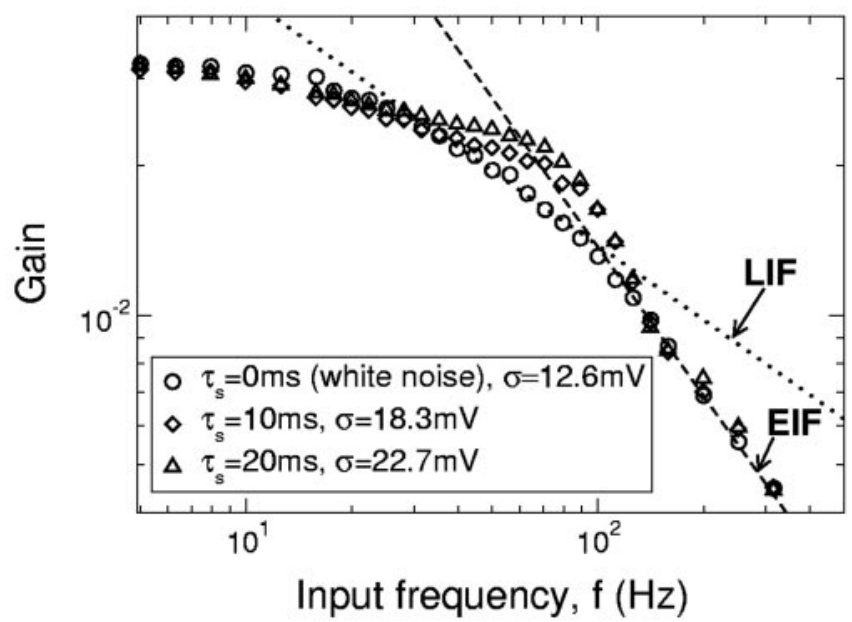

Figure 6. Influence of noise correlation time on the gain of the EIF neuron. The stationary firing rate is $\nu_{0}=30 \mathrm{~Hz}$, and the noise level is adjusted so that the low-frequency response is constant as the synaptic decay time, $\tau_{s}$, is varied from $0 \mathrm{msec}$ (white noise) to $20 \mathrm{msec}$.
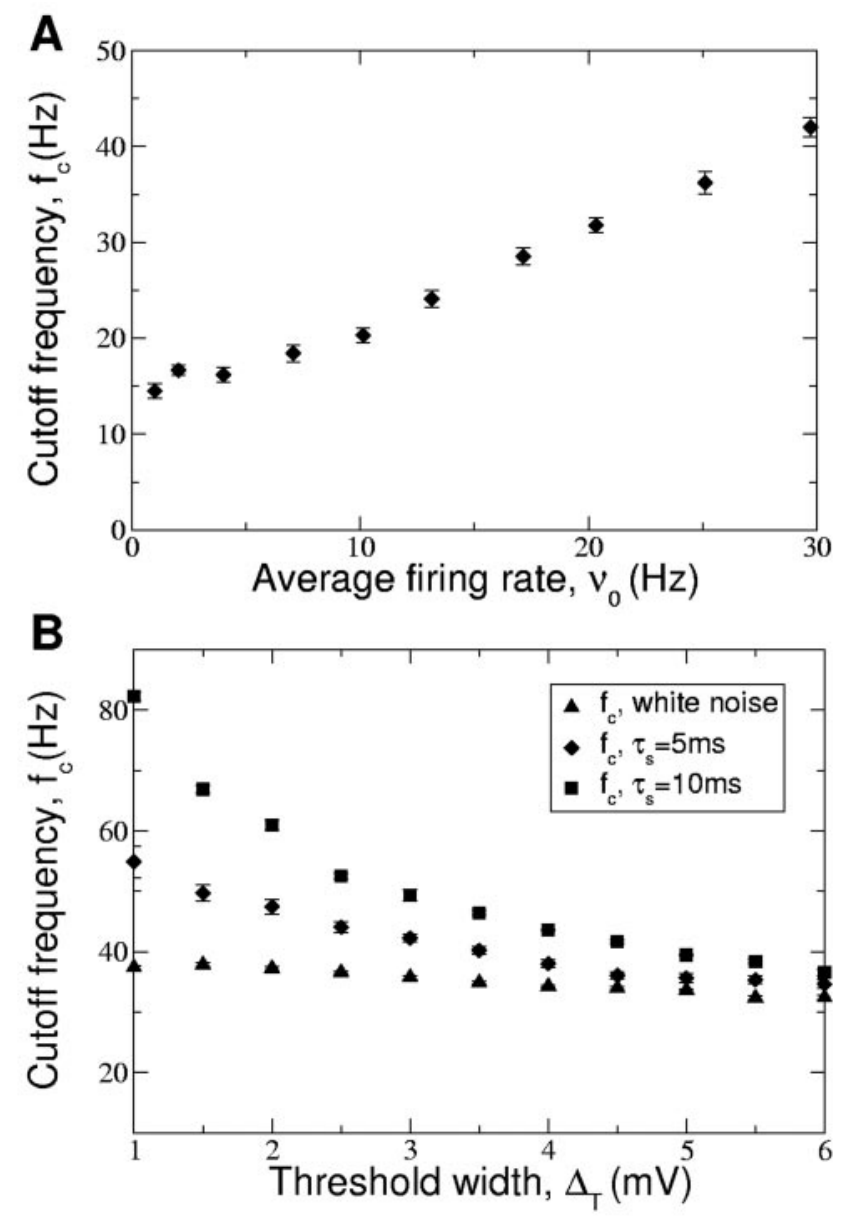

Figure 7. $A, B$, Cutoff frequency of the ElF filter in the high-noise regime as a function of the firing rate $(A)$ and spike slope factor $(B)$. In $A$, the parameters of the EIF model are chosen to match the WB model $\left(\Delta_{T}=3.48 \mathrm{mV}\right) . \operatorname{In} B, \delta_{T}$ is varied. In both panels, $\sigma=8 \mathrm{mV}$. $A$, The cutoff frequency is approximately proportional to the average firing rate $\nu_{0}$ (simulations with white noise). $B$, The cutoff frequency depends weakly on the slope factor $\Delta_{T}$ for white noise but strongly increases when $\delta_{T}$ decreases for colored noise $\left(\nu_{0}=24 \mathrm{~Hz}\right.$; values of the synaptic time constants are indicated in the legend).

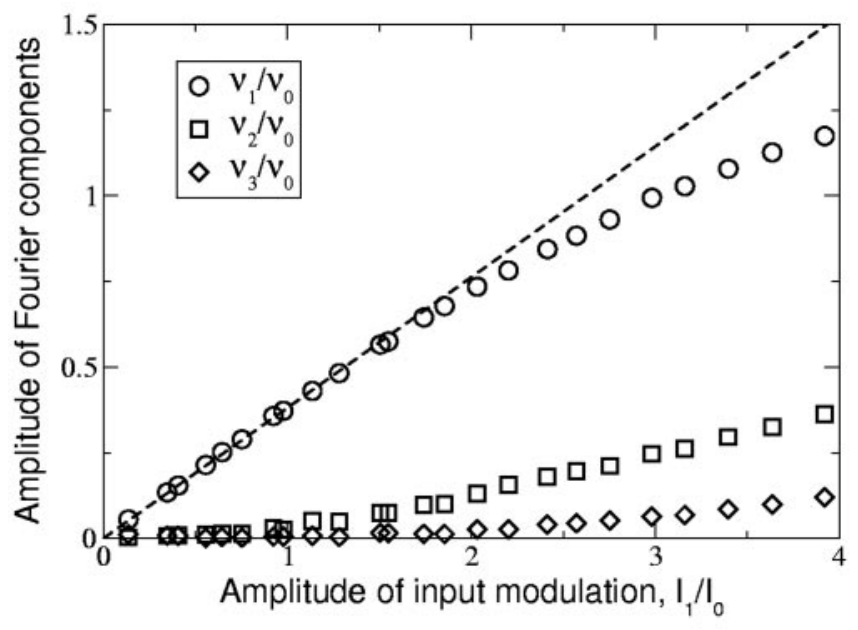

Figure 8. Amplitude of the first three Fourier components of the response, normalized by the average firing rate, $\nu_{0}$, as a function of the strength of input modulation. Circles, First Fourier component; squares, second Fourier component; diamonds, third Fourier component. Parameters: $\sigma=6.3 \mathrm{mV} ; \nu_{0}=20 \mathrm{~Hz} ; f=20 \mathrm{~Hz}$. The first Fourier component, $\nu_{1}$, is linear in $l_{1}$ up to modulations of the firing rate of order $\nu_{0}$. The higher Fourier components are negligible up to approximately the same amount of modulation. Note that all simulations presented in this study (except in this figure) are done with $\nu_{1} / \nu_{0} \approx 0.25$, well into the domain of validity of the linear approximation.

\section{Domain of validity of the linear response}

In the previous sections, we analytically computed the linear response of the EIF neuron to modulated input in the limit of small or large modulation frequency, $f$. These results should provide a correct quantitative description of the neuron dynamics in these limits for sufficiently weak amplitude modulation, $I_{1}$. The numerical simulations presented above, performed for a finite but small value of $I_{1}$, confirm this expectation. They also extend our study by investigating the neuronal linear filter at intermediate values of the input frequency.

How large can the input modulation be for the response of the neuron to still be linear? To address this issue, we performed simulations at various levels of input modulation and measured the amplitude of the Fourier components of the response up to order 3. Results of these simulations are displayed in Figure 8 for $\sigma=6.3 \mathrm{mV}, \nu_{0}=20 \mathrm{~Hz}$, and an input modulation frequency of $f=20 \mathrm{~Hz}$. For these parameters, $\nu_{1}$ varies linearly with $I_{1}$, and higher order Fourier components are negligible up to input modulations that induce temporal variations of the firing rate as large as $75 \%$ of the average firing rate. This shows that the neuron responds in a linear manner in this range of firing rate variations. A similar range of validity was obtained for all values of $f$ tested $(0-100 \mathrm{~Hz})$ in the strong noise regime (the boundaries of strong and low-noise regimes are indicated in Fig. 4). In the low-noise regime, the domain of validity is on the same order of magnitude, except for frequencies close to resonances.

\section{Comparison of the linear responses of EIF and conductance-based neurons}

In Figure 9, we compare the gain and phase shifts of the EIF and WB models for two levels of noise, $\sigma$, and average firing rates, $\nu_{0}$. We found a remarkable agreement for the gain of the two models in all ranges of frequencies studied. For the phase shift, the agreement is also excellent but in a smaller range of frequencies, up to $100 \mathrm{~Hz}$. Above $100 \mathrm{~Hz}$, the phase lag is significantly smaller in the EIF model than in the WB model. This is a consequence of the 

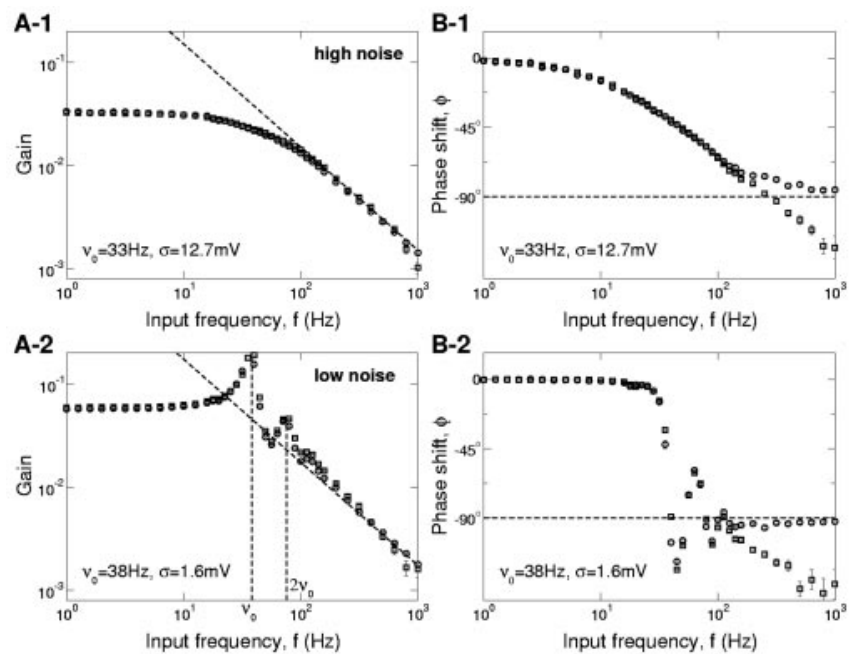

Figure 9. Comparison of the filters of the WB model $(\square)$ and the EIF model $(O)$. We plot the gain $(A, 1-2)$ and phase shift $(B, 1-2)$ with high $(A-1, B-1)$ and low noise $(A-2, B-2)$. Note the good agreement in all regimes for the gain. The phase shifts of both models are very similar up to an input frequency at $\sim 100 \mathrm{~Hz}$ in which the WB model has an additional phase lag. This is a consequence of the fixed delay between EIF and WB spike time shown in Figure $3 B$.

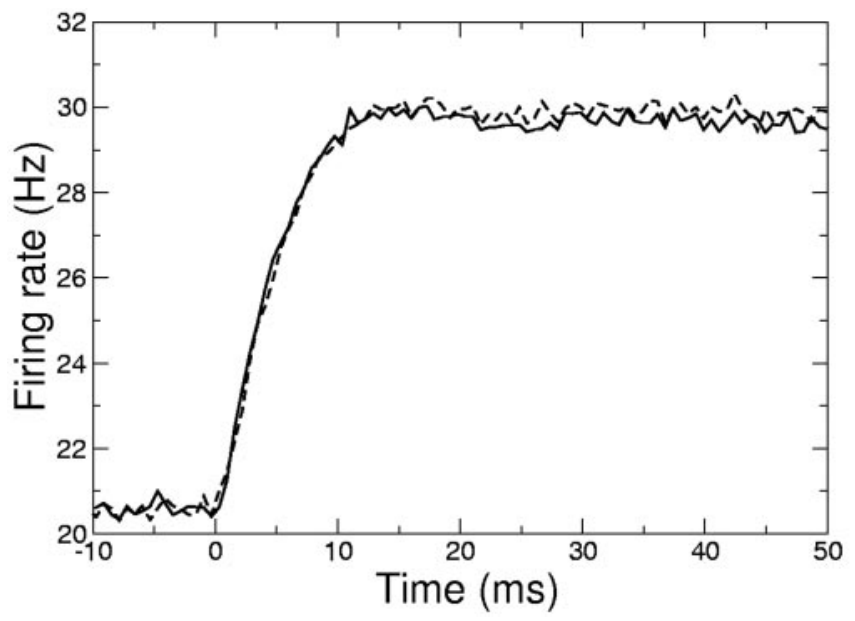

Figure 10. Comparison of the response of the WB (solid line) and EIF (dashed line) models to a current step (average, $>1,600,000$ repetitions; firing rate computed in $0.6 \mathrm{msec}$ bins; $\sigma=$ $6.3 \mathrm{mV}$ ). The time course of the responses of WB and EIF models is indistinguishable. The agreement between both models is excellent, confirming the close match of the linear filters shown in Figure 9.

delay $\delta \approx 0.2$ msec between the spikes in the EIF and WB models, which is clearly visible in Figure $3 B$. The corresponding additional phase lag is $2 \pi f \delta$. This phase shift depends on the voltage $V_{s}$, which is used for the definition of the spike times in the WB model.

In Figure 10, we show how the instantaneous firing rate of $\mathrm{WB}$ and EIF models responds to a current step that brings the neuron from 20 to $30 \mathrm{~Hz}$. Again, the figure shows an excellent agreement of EIF and WB model responses, as expected from Figures 8 and 9.

Effect of sodium channel activation kinetics on high-frequency behavior

Sodium channels have a voltage-dependent activation time constant on the order of $0.1 \mathrm{msec}$ (Martina and Jonas, 1997). The WB model makes the simplifying assumption that the sodium activa-

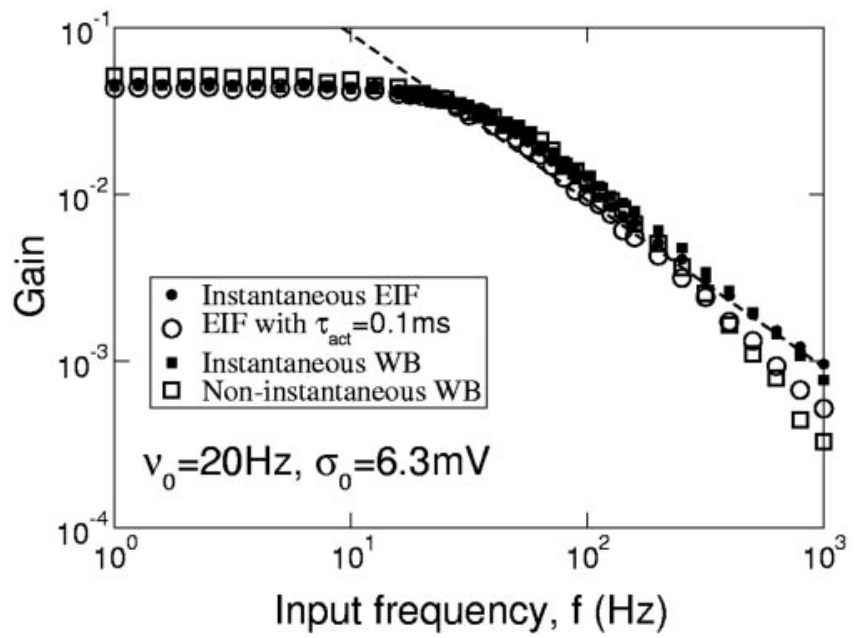

Figure 11. Gain of the firing rate modulation of EIF and WB models with noninstantaneous sodium activation kinetics. The dashed line shows the asymptotic regime for an instantaneous activation. The activation kinetics attenuates the response slightly more only for frequencies beyond several hundred Hertz.

tion is instantaneous. Because $\psi(V)$ depends on the voltage directly in the nonlinear IF models, the effective spike initiation is also instantaneous in these models. In most situations, this assumption will not affect the results much, but there is clearly a frequency above which the finite activation time constant will become important.

To measure at which frequency the activation kinetics affects the firing rate modulation, we introduced the activation kinetics in the WB model as in the Hodgkin-Huxley formalism. For the EIF model, we replaced the spike-generating current, $\psi(V)$, by a dynamical variable with an activation time constant, $\tau_{\text {act }}=0.1$ msec: $\tau_{\text {act }} d \psi / d t=-\psi+g_{L} \Delta T \exp \left[\left(V-V_{T}\right) / \Delta_{T}\right]$. After each spike, the potential was reset to $V_{r}$, and the spike-generating current was reset to $\psi\left(V_{r}\right)$. Results are shown in Figure 11. In both models, the firing rate modulation was not affected at low and intermediate frequencies. It was only slightly more attenuated and had a larger phase lag at input frequency beyond several hundred Hertz. In particular, the cutoff frequency was not affected, and the instantaneous model was sufficient to describe the neuron dynamics in a wide range of input frequencies $(0-500$ $\mathrm{Hz}$ ). Slower activation kinetics decreased the frequency at which the deviation from the $1 / f$ behavior occurred. For example, for time constants of $1 \mathrm{msec}$, the response is already significantly more attenuated than $1 / f$ below $100 \mathrm{~Hz}$.

\section{High-frequency behavior of other}

conductance-based neurons

So far, our results suggest that the high-frequency response depends only on the properties of the current leading to spike generation. To test this prediction, we performed numerical simulations of a conductance-based model with the same fast sodium current as in the WB model but with several additional ionic currents (Hansel and van Vreeswijk, 2002) (see Materials and Methods). These additional currents modify the subthreshold behavior of the neuron and act on time scales slower than the sodium activation time. They have a significant effect on the response at low-input frequencies because of, in particular, the adaptation current. However, Figure 12 shows that, above the cutoff frequency, the amplitude of the firing rate modulation decreases again as $1 / f$ as in the EIF model. The dashed line in 
A

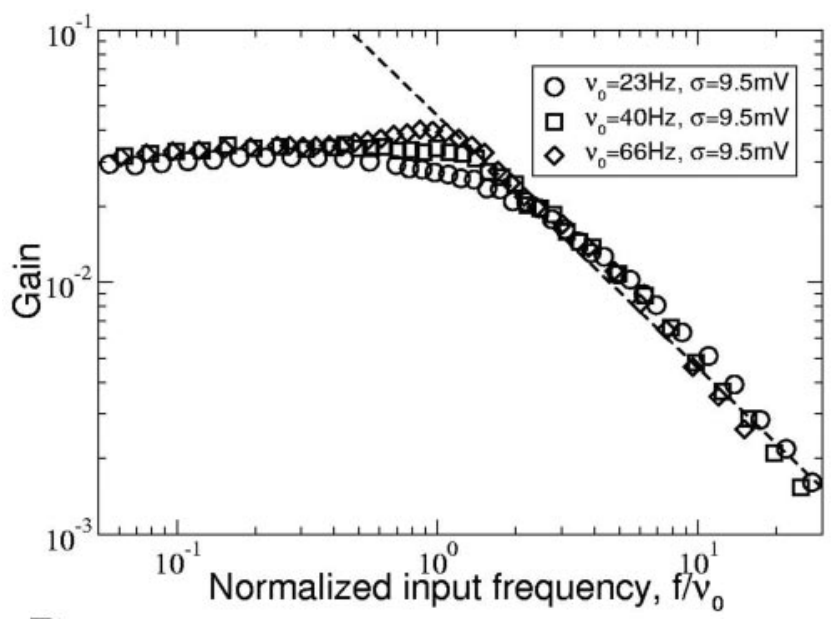

B

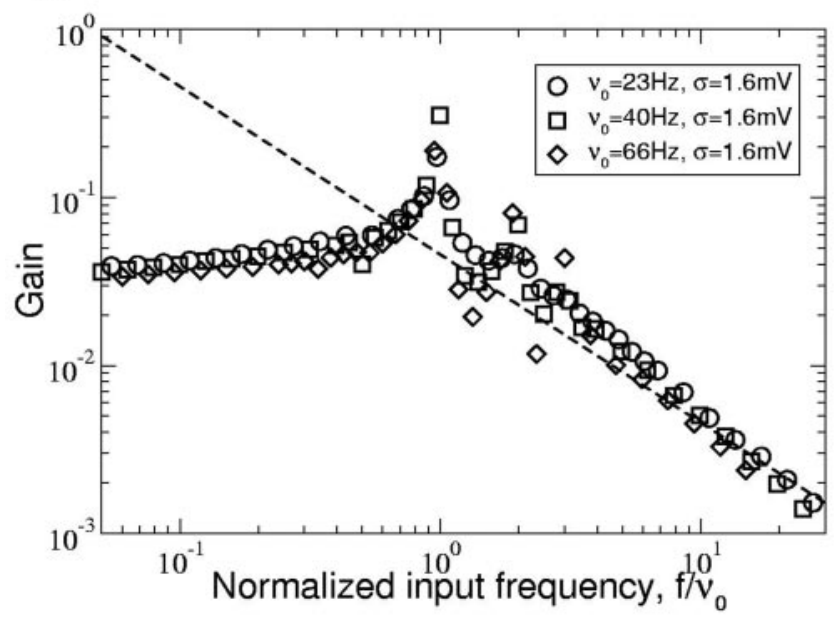

Figure 12. Amplitude of the firing rate modulation for a conductance-based model with additional currents. $A, B$, Response to inputs with strong $(A)$ and weak $(B)$ noise and various average firing rates (indicated in corresponding panels). The high frequency behavior is proportional to $1 / f$ with a cutoff close to the average firing rate, $\nu_{0}$. The dashed line shows the EIF high-input frequency asymptotic regime with parameters matching the sodium activation curve of the conductance-based neuron. Note that in this figure, the frequency is normalized to the average firing rate, $v_{0}$, so that all response curves match approximately at high frequencies. The $1 /$ f regime extends up to $1000 \mathrm{~Hz}$ as in the WB model.

Figure 12 shows the amplitude in the asymptotic regime predicted by the EIF neuron (Eq. 15) with the same values of $C$ and $\Delta_{T}$ as for the regularly firing and WB neurons. This confirms that high-input frequency behavior is determined only by intrinsic currents leading to spike emission and the average firing rate.

\section{Discussion}

We studied how the firing rate response of a neuron to an oscillating input depends on the modulation frequency, $f$. The models that we investigated include conductance-based models as well as generalized integrate-and-fire models. For sufficiently strong noise, we found that these neurons behave like a low-pass filter. For $f$ below a cutoff frequency $f_{c}$, the response modulation is weakly dependent on $f$. For $f>f_{c}$, it decreases rapidly with $f$ and behaves at large $f$ like a power law $C / f^{\alpha}$, where $C$ is a prefactor independent of $f$. We analytically calculated $\alpha$ and $C$ for generalized integrate-and-fire models and have shown that $\alpha$ depends on the nonlinearity of spike initiation.
Of particular interest is the case of neurons with an exponential spike nonlinearity (EIF model). In this case, $\alpha=1$, and $C$ depends on both the average firing rate of the neuron and the sharpness of spike initiation characterized by the spike slope factor, $\Delta_{T}$. This high-frequency behavior is independent of the properties of the noise; it holds for white noise as well as colored noise and for current-based and conductance-based noise. In contrast, all other types of nonlinearity lead to exponents $\alpha$ different from 1.

Fast sodium currents involved in action potentials increase exponentially near firing onset. Therefore, we expect that our analytical results derived in the framework of the EIF are relevant to predicting the response of real neurons to rapidly varying inputs. This is further supported by our simulations of conductance-based models, the quantitative behavior of which is identical to that found for the EIF.

We have also shown that the cutoff frequency of the EIF neuron increases approximately linearly with the average firing rate, and that it is a decreasing function of the spike slope factor $\Delta_{T}$. The latter dependency is very mild for white noise but much more pronounced for colored noise. We expect this result to be generic, as confirmed by our simulations of simple conductancebased models.

\section{Implications for modeling}

We have shown how a simple conductance-based neuronal model can be reduced in a systematic way to a new type of integrate-and-fire model, the EIF model, in which the dynamics involve a current that increases exponentially with the membrane potential. Remarkably, the responses to rapidly fluctuating inputs of the full and reduced models are indistinguishable. More generally, the filter characteristics are quantitatively similar for the two models in the entire range of input frequencies. This is in contrast to the LIF model, the response properties (Gerstner, 2000; Brunel et al., 2001; Fourcaud and Brunel, 2002; Mazurek and Shadlen, 2002; van Rossum et al., 2002) of which differ qualitatively from those of conductance-based models at all frequencies, as shown here. Also, although QIF and conductance-based neurons behave similarly at low frequencies, qualitative discrepancies remain at high frequencies.

The stability of asynchronous firing in large networks of interacting neurons has been studied extensively in LIF (Abbott and van Vreeswijk, 1993; Treves, 1993; Brunel and Hakim, 1999; Brunel, 2000) and QIF (Hansel and Mato, 2001, 2003) neuronal networks. The approach developed in these studies can be generalized to some extent for neurons with more realistic dynamics, provided that one knows how they respond to weakly modulated oscillating inputs. Hence, the present study paves the way for a greater understanding of the synchronization properties of conductance-based neurons. In particular, the presence of a cutoff frequency in the filtering properties of conductance-based neurons indicates that there is an upper bound to the frequency of population oscillations, which can emerge in large networks (Geisler et al., 2002).

\section{Functional implications}

Our results can be used to determine how many neurons are needed to detect a transient signal in noisy conditions, given single-neuron characteristics. Equivalently, one can calculate the minimal duration a given signal should last to be detected. Consider, for example, a population of 100 neurons emitting at a background rate of $10 \mathrm{~Hz}$ and subjected to a transient signal that, 
if applied for a time long enough, would increase the firing rate to $20 \mathrm{~Hz}$. An ideal detector must be able to distinguish the transient increase of the population rate induced by the signal from rate fluctuations attributable to the finite number of neurons. This can be done using a receiver operator characteristics analysis (Green and Swets, 1966). Briefly, one computes the distributions of the spike counts in a given interval under two conditions (signal or no signal). One then computes the probability of correct detection of the signal (i.e., the probability that the spike count is larger when the signal is present than when it is absent). If neurons have very sharp spikes $\left(\Delta_{T}\right.$ close to zero) in the presence of correlated noise, the signal can be detected in $\sim 5 \mathrm{msec}$ with $90 \%$ accuracy. If the spike slope factor is $\Delta_{T}=3 \mathrm{mV}$, the signal can be detected only in $\sim 12 \mathrm{msec}$ with the same accuracy. The detection time increases further for larger values of $\Delta_{T}$.

\section{Experimental implications}

How sharp are spikes in cortical neurons?

Our work shows that the spike slope factor, $\Delta_{T}$, is one of the main parameters on which the response of a neuron to fluctuating inputs depends. Activation curves of $\mathrm{Na}^{+}$channels have been measured in several preparations, including neocortical pyramidal cells (Fleidervish et al., 1996), hippocampal pyramidal cells, granule cells, and basket cells (Martina and Jonas, 1997; Fricker et al., 1999; Ellerkmann et al., 2001). These authors used Boltzmann functions to fit the observed data. Using their best-fit parameters, one finds $\Delta_{T}$ in the range of 3-6 $\mathrm{mV}$ for these types of cells. However, in all cases, there are few data points in the region of the threshold, leading to a considerable uncertainty in the estimate of this parameter. Therefore, more experiments are needed to determine the spike slope factor of cortical neurons.

Experimental measurements of the linear response of neurons The instantaneous firing rate of neurons responding to sinusoidal currents has been measured in slice preparations (Knight, 1972b; Carandini et al., 1996; Chance, 2000). Carandini et al. (1996) and Chance (2000) found cutoff frequencies in the range of $10-100 \mathrm{~Hz}$ that increase with the average firing rate. This is consistent with our findings. However, none of these studies have systematically explored high-frequency behavior. Bair and Koch (1996) measured the poststimulus time histogram power spectra

Table 2. Gating variables of conductance-based models

\begin{tabular}{|c|c|c|}
\hline$x$ & $a_{x}$ & $b_{x}$ \\
\hline$m$ & $\frac{0.1(\mathrm{~V}+35)}{1-\exp [-0.1(\mathrm{~V}+35)]}$ & \multirow{2}{*}{$\begin{array}{c}4 \exp [-(V+60) / 18] \\
5\end{array}$} \\
\hline$h$ & $0.35 \exp [-(V+58) / 20]$ & \\
\hline$n$ & $\frac{0.05(V+34)}{1-\exp [-0.1(V+34)]}$ & $0.625 \exp [-V+44) / 80]$ \\
\hline$x$ & $x_{\infty}$ & $\tau_{\mathrm{x}}$ \\
\hline$a$ & $\frac{1}{1+\exp [-(V+50) / 20]}$ & Instantaneous \\
\hline$b$ & $\overline{1+\exp [(V+80) / 6]}$ & 20 \\
\hline$S$ & $\begin{array}{c}1+\exp [-(v+40) / 5] \\
1\end{array}$ & Instantaneous \\
\hline$Z$ & $\overline{1+\exp [-0.7(\mathrm{~V}+30)]}$ & 50 \\
\hline
\end{tabular}

Table 3. Conductance density in $\mathrm{mS} / \mathrm{cm}^{2}$ and reversal potentials in $\mathrm{mV}$ for the ionic channels in the conductance-based models

\begin{tabular}{lll}
\hline$x$ & $g_{x}$ & $V_{x}$ \\
\hline $\mathrm{Na}$ & 35 & 55 \\
$\mathrm{NaP}$ & 0.08 & 55 \\
$K$ & 15 & -90 \\
$A$ & 2.5 & -90 \\
$K_{s}$ & 0.5 & -90 \\
\hline
\end{tabular}

of MT neurons in response to visual stimuli consisting of randomly moving dots. In the examples shown in this study, the output power spectrum is rapidly attenuated in the $30-100 \mathrm{~Hz}$ frequency range. However, it is difficult to interpret these results, because the frequency content of the synaptic input to MT neurons is unknown. Thus, additional experiments are needed to test our prediction for the $1 / f$ attenuation of the response at high frequencies.

\section{Testing our experimental predictions}

The main predictions of our work are: (1) the neuronal gain decays as $1 / f$ at high frequency, independently of the characteristics of the input, (2) the cutoff frequency increases with the average firing rate, and (3) the cutoff frequency increases when the spike slope factor decreases in the presence of temporally correlated noise. One possible experimental test of these results would be to study the response of neurons in vitro, in which sodium channels underlying spike initiation are blocked with TTX and replaced, using dynamic clamp techniques by an artificial sodium current with known properties. Note that because the dynamics of sodium currents underlying spike initiation are fast, a dedicated analog circuit may be needed to emulate them with the dynamic clamp method. A similar experiment can be devised in vivo (e.g., in cortical neurons). Using QX314 (lidocaine $N$-ethyl bromide quaternary salt) intracellularly, sodium currents in a specific cell can be blocked without affecting the activity of its neighboring cells. This would preserve the background noise that the cell receives from the rest of the network and allow for the study of its effect on the response.

\section{Appendix}

\section{A. Conductance-based models}

The Wang-Buszáki model was introduced by Wang and Buzsáki (1996). The membrane potential $V(t)$ is governed by the following equation:

$$
C_{M} \frac{d V}{d t}=-I_{L}-I_{\mathrm{Na}}-I_{K}+I_{\mathrm{syn}}(t)
$$

where $C_{M}$ is the membrane capacitance $\left(C_{M}=1 \mu \mathrm{F} / \mathrm{cm}^{2}\right), I_{L}=$ $g_{L}\left(V-V_{L}\right)$ is the leak current $\left(g_{L}=0.1 \mathrm{mS} / \mathrm{cm}^{2} ; V_{L}=-65 \mathrm{mV}\right.$; $\left.\tau_{m}=C / g_{L}=10 \mathrm{msec}\right), I_{\mathrm{Na}}=g_{\mathrm{Na}} m^{3} h\left(V-V_{\mathrm{Na}}\right)$ is the sodium Hodgkin-Huxley current with an instantaneous activation variable, $I_{K}=g_{K} n^{4}\left(V-V_{K}\right)$ is the delayed rectifier potassium current, and $I_{\text {syn }}(t)$ is the input current defined in Materials and Methods.

The dynamical equations for the gating variables are:

$$
\frac{d x}{d t}=\frac{x_{\infty}(V)-x}{\tau_{x}(V)},
$$

where $x=m, h, n$. All functions $x_{\infty}(V), \tau_{x}$ are given in Table 2 . The respective maximum conductance densities and the reversal potentials of the ionic currents are given in Table 3.

Another conductance-based model used in this work is taken 
from Hansel and van Vreeswijk (2002). The dynamics of the model are described by:

$$
C_{M} \frac{d V}{d t}=-I_{L}-I_{\mathrm{Na}}-I_{\mathrm{Na} P}-I_{K}-I_{A}-I_{K s}+I_{\mathrm{syn}},
$$

where the currents $I_{L}, I_{\mathrm{Na}}$, and $I_{K}$ are identical to the WB model (except $V_{L}=-70 \mathrm{mV}$ ), but three additional ionic currents are present: (1) a persistent sodium current, $I_{\mathrm{Na} P}=g_{\mathrm{Na} P} s_{\infty}(V)(V-$ $\left.V_{\mathrm{Na}}\right)$, (2) an A-type potassium current, $I_{A}=g_{A} a_{\infty}(V)^{3} b\left(V-V_{K}\right)$, and (3) a slow potassium current responsible for spike adaptation, $I_{K s}=g_{K s} z\left(V-V_{K}\right)$.

The dynamical equations for the gating variables $m, h, n, b$, and $z$ are given by Equation 16. All gating variable parameters are given in Table 2 . The respective maximum conductance densities and the reversal potentials of the ionic currents are given in Table 3.

In most of this study, we neglect the sodium activation kinetics, and we take $m=m_{\infty}$.

\section{B. Analytical calculations of the neuronal response to sinusoidal modulated noisy inputs}

B1. Gaussian white noise

We rewrite Equation 6 as:

$$
C \frac{d V}{d t}=g_{L} F(V)+I_{\text {syn }}(t)
$$

with $F(V)=-V+V_{L}+\psi(V) / g_{L}$ and:

$$
\begin{gathered}
I_{\text {syn }}(t)=I(t)+\sigma \sqrt{C g_{L}} \eta(t), \\
I(t)=I_{0}+I_{1} \cos (\omega t),
\end{gathered}
$$

where $I(t)$ is the deterministic part of the input current, and $\eta(t)$ is a Gaussian white noise with zero mean and unitary SD. The frequency of the modulation is $f$ and $\omega \equiv 2 \pi f$. For convenience, we also define $\mu(t)=I(t) / g_{L}$, which can be written as $\mu(t)=\mu_{0}+$ $\mu_{1} \cos (\omega t)$, with $\mu_{0}=I_{0} / g_{L}, \mu_{1}=I_{1} / g_{L}$. We denote the membrane time constant $\tau_{m}=C / g_{L}$. Equation 18 can then be rewritten as:

$$
\tau_{m} \frac{d V}{d t}=F(V)+\mu(t)+\sigma \sqrt{\tau_{m}} \eta(t)
$$

The distribution of voltages at time $t$ obeys the Fokker-Planck equation (Risken, 1984):

$$
\tau_{m} \frac{\partial P}{\partial t}=\frac{\sigma^{2}}{2} \frac{\partial^{2} P}{\partial V^{2}}-\frac{\partial}{\partial V}[F(V)+\mu(t)] P,
$$

with the boundary conditions:

$$
\lim _{V \rightarrow+\infty} J_{V}(V, t)=\nu(t),
$$

where

$$
J_{V}(V, t)=\frac{[F(V)+\mu(t)]}{\tau_{m}} P(V, t)-\frac{\sigma^{2}}{2 \tau_{m}} \frac{d P}{d V}(V, t) .
$$

The reset of the voltage to $V_{r}$ after the spike is taken into account by requiring that:

$$
J_{V}\left(V_{r}^{+}, t\right)=J_{V}\left(V_{r}^{-}, t\right)+\nu(t) .
$$

We solve this equation assuming that $\mu_{1} \ll \mu_{0}$. For the sake of simplicity, we use complex notations and write to first order:

$$
\begin{aligned}
P(V, t) & =P_{0}(V)+P_{1}(V, \omega) e^{i \omega t}, \\
\nu(t) & =\nu_{0}+\nu_{1}(\omega) e^{i \omega t}, \\
J_{v}(V, t) & =J_{V, 0}(V)+J_{V, 1}(V, \omega) e^{i \omega t} .
\end{aligned}
$$

where $P_{1}(V, \omega), \nu_{1}(\omega)$, and $J_{V, 1}(V, \omega)$ are complex quantities, $\left|P_{1}\right| \ll P_{0},\left|\nu_{1}\right| \ll \nu_{0},\left|J_{V, 1}\right| \ll J_{V, 0}$.

Substituting Equation 24 in Equation 22 and keeping only the terms at leading order, one finds that $P_{0}(V)$ satisfies the equation:

$$
\frac{\sigma^{2}}{2} \frac{d^{2} P_{0}}{d V^{2}}-\frac{d}{d V}\left[F(V)+\mu_{0}\right] P_{0}(V)=0,
$$

which, after one integration over $V$, gives:

$$
\frac{\sigma^{2}}{2} \frac{\partial P_{0}}{\partial V}-\left[F(V)+\mu_{0}\right] P_{0}=\left\{\begin{array}{c}
K_{+} \text {for } V>V_{\mathrm{r}} \\
\mathrm{K}_{-} \text {for } V<V_{r}
\end{array}\right\},
$$

where $K_{+}$and $K_{-}$are constants. The boundary condition (Eq. 12) and reset (Eq. 23) determine $K_{+}$and $K_{-}$:

$$
K_{+}=-\nu_{0} \tau_{m}, \quad K_{-}=0 .
$$

The solution of Equation 26 is:

$$
P_{0}(V)=\frac{2 \nu_{0} \tau_{m}}{\sigma^{2}} \int_{\max \left(V, V_{r}\right)}^{+\infty} \exp \left(-\frac{2}{\sigma^{2}} \int_{V}^{u}\left[F(x)+\mu_{0}\right] d x\right) d u
$$

The normalization of the distribution $P_{0}(V)$ determines the average firing rate, $\nu_{0}$. One finds:

$$
\nu_{0}=\left\{\frac{2 \tau_{m}}{\sigma_{0}^{2}} \int_{-\infty}^{+\infty} d V\left[\int_{\max \left(V, V_{r}\right)}^{+\infty}\left(\left[F(x)+\mu_{0}\right] d x\right) d u\right]\right\}^{-1} .
$$

We now consider the first order contributions in the expansions of Equation 22. One finds:

$$
i \omega \tau_{m} P_{1}=\frac{\sigma^{2}}{2} \frac{\partial^{2} P_{1}}{\partial V^{2}}-\frac{\partial}{\partial V}\left(F+\mu_{0}\right) P_{1}-\mu_{1} \frac{\partial P_{0}}{\partial V} .
$$

The boundary condition at the same order gives:

$$
\begin{aligned}
\nu_{1}(\omega) & =\lim _{V \rightarrow+\infty}\left[\frac{F(V)+\mu_{0}}{\tau_{m}} P_{1}(V, \omega)\right. \\
& \left.-\frac{\sigma^{2}}{2 \tau_{m}} \frac{\partial P_{1}}{\partial V}(V, \omega)+\frac{\mu_{1}}{\tau_{m}} P_{0}(V)\right], \\
& =\lim _{V \rightarrow+\infty}\left[\frac{F(V)}{\tau_{m}} P_{1}(V, \omega)\right] .
\end{aligned}
$$

In the last equality, we used the fact that $F(V)$ goes to infinity at large $V$.

The solution of Equations 30 and 31 simplifies in two limits: 


\section{Low-input frequency}

At low frequencies $\left(\omega \tau_{m} \ll 1\right), P(V, t)$ follows adiabatically the slowly changing input, and the left-hand side of Equation 30 can be neglected. It is then easy to check that $P_{1}=\mu_{1}\left(\partial P_{0} / \partial \mu\right)$ is a solution of Equation 30. Using $P_{0}(V) \approx{ }_{\mathrm{V} \rightarrow \infty} \nu_{0} / F(V)$ (Eqs. 26, 31 ), we can compute the firing rate modulation in the low-input frequency limit:

$$
\nu_{1}(\omega \rightarrow 0)=\mu_{1} \frac{\partial \nu_{0}}{\partial \mu}
$$

\section{High-input frequency}

Expanding Equation 30 in powers of $1 / \omega$ and keeping only the leading order gives:

$$
P_{1}(V)=-\frac{\mu_{1}}{i \omega \tau_{m}} \frac{\partial P_{0}}{\partial V}(V)+\mathrm{O}\left(\frac{1}{\omega^{2}}\right) .
$$

and $\nu_{1}$ is determined by the large $V$ behavior of $P_{1}(V)$ (Eq. 29). Using Equation 26 and the fact that $F(V)$ diverges at large $V$, one finds:

$$
P_{0}(V) \approx \frac{\nu_{0}}{F(V)}
$$

and thus

$$
\frac{\partial P_{0}}{\partial V} \approx-\frac{\nu_{0} F^{\prime}(V)}{F^{2}(V)}
$$

Inserting Equation 35 into Equation 33, we find:

$$
P_{1} \approx \frac{\mu_{1} \nu_{0}}{i \omega \tau_{m}} \frac{F^{\prime}(V)}{F^{2}(V)} .
$$

Finally, inserting Equation 36 into Equation 31, we find:

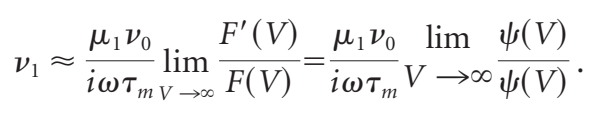

This formula can be applied to the EIF model to show that $\nu_{1}$ decays like $1 / \omega$ at large $\omega$. In contrast, for any polynomial $F(V)$, the $1 / \omega$ term vanishes, and it is necessary to calculate $P_{1}$ and $\nu_{1}$ at the next order (i.e., $1 / \omega^{2}$ ). One finds that $\nu_{1}=A / \omega^{2}$, where the prefactor $A$ is proportional to $\lim _{V \rightarrow \infty}\left(F^{\prime 2}-F F^{\prime \prime}\right) / F$. In particular, if $F \approx V^{2}$ at large $V, A$ is finite, and $\nu_{1}$ decays like $1 / \omega^{2}$ at large $\omega$. This is the case for the QIF model for which one finds:

$$
\nu_{1}(\omega)=-\frac{\nu_{0} \mu_{1}}{\Delta_{T}\left(\omega \tau_{m}\right)^{2}} .
$$

If $F \approx V^{k}>2$ at large $V, A$ diverges. In this case, one expects the firing rate modulation to decrease like $1 / \omega^{\alpha}$ with $1<\alpha<2$.

\section{B2. Temporally correlated noise}

In the previous section, we assumed white Gaussian input noise. Here, we show that the presence of correlations does not affect the high-frequency behavior of the spike response modulation of the EIF neurons. Assuming that synaptic current decays expo- nentially with a synaptic time constant, $\tau_{s}$, the equations for the dynamics of the IF neurons are:

$$
\begin{gathered}
\tau_{m} \frac{d V}{d t}=F(V)+\mu_{\text {syn }}(t)+\mu_{\text {ext }}(t), \\
\tau_{s} \frac{d \mu_{\text {syn }}}{d t}=-\mu_{\text {syn }}+\mu_{0}+\sigma \sqrt{\tau_{m}} \eta(t) .
\end{gathered}
$$

The probability density function, $P\left(V, \mu_{\text {syn }}, t\right)$ (Brunel et al., 2001; Haskell et al., 2001; Fourcaud and Brunel, 2002), satisfies a twodimensional Fokker-Planck equation:

$$
\begin{aligned}
\frac{\partial P}{\partial t}=\frac{1}{\tau_{s}}\left[\frac{\sigma^{2} \tau_{m}}{2 \tau_{s}} \frac{\partial^{2} P}{\partial \mu_{\mathrm{syn}}^{2}}+\right. & \left.\frac{\partial}{\partial \mu_{\mathrm{syn}}}\left(\mu_{\mathrm{syn}}-\mu_{0}\right) P\right] \\
& -\frac{1}{\tau_{m}} \frac{\partial}{\partial V}\left(\left[\mu_{\mathrm{syn}}+\mu_{\mathrm{ext}}+F(V)\right] P\right) .
\end{aligned}
$$

The probability flux is now a two-dimensional vector with components:

$$
\begin{gathered}
J_{V}=\frac{\mu_{\text {syn }}+\mu_{\text {ext }}+F(V)}{\tau_{m}} P, \\
J_{\mu_{\text {syn }}}=-\left(\frac{\mu_{\text {syn }}-\mu_{0}}{\tau_{s}}\right) P+\frac{\sigma^{2} \tau_{m}}{2 \tau_{s}^{2}} \frac{\partial P}{\partial \mu_{\text {syn }}} .
\end{gathered}
$$

In particular, in the large $V$ limit:

$$
P\left(V, \mu_{\text {syn }}, t\right) \sim_{V \rightarrow+\infty} \frac{1}{F(V)}\left[J_{\mathrm{V}}\left(V, \mu_{\mathrm{syn}}, t\right)\right],
$$

because $F(V)$ diverges at large $V$. As before, the instantaneous firing rate is given by:

$$
\nu(t)=\lim _{V \rightarrow \infty} \int_{-\infty}^{+\infty} J_{V}\left(V, \mu_{\text {syn }}, t\right) d \mu_{\text {syn }} .
$$

To solve these equations, one expands $P\left(V, \mu_{\text {syn }}, t\right), J_{V}\left(V, \mu_{\text {syn }}, t\right)$ and $\nu(t)$ in a way similar to the case of white noise. One finds:

$$
\nu_{\kappa}=\lim _{V \rightarrow \infty} \int_{-\infty}^{+\infty} J_{V, \kappa}\left(V, \mu_{\mathrm{syn}}\right) d \mu_{\mathrm{syn}},
$$

and

$$
P_{\kappa}\left(V, \mu_{\text {syn }}\right) \sim_{V \rightarrow+\infty} \frac{1}{F(V)}\left[J_{V, \kappa}\left(V, \mu_{\text {syn }}\right)\right]
$$

for $k=0,1$. A similar analysis, as in the white noise case, shows that at first order in $1 / \omega$ :

$$
P_{1}=-\frac{\mu_{1}}{i \omega \tau_{m}} \frac{\partial P_{0}}{\partial V}
$$

Combining Equations 44-45, one can show that:

$$
\begin{aligned}
\nu 1 & \sim{ }_{\omega \rightarrow \infty}-\frac{\mu_{1}}{i \omega \tau_{m}} \lim _{V \rightarrow \infty} \int_{-\infty}^{+\infty} F(V) \frac{\partial P_{0}}{\partial V} d \mu_{\text {syn }}, \\
& \sim{ }_{\omega \rightarrow \infty} \frac{\mu_{1} \nu_{0}}{i \omega \tau_{m}} \lim _{V \rightarrow \infty} \frac{F(V)}{F(V)}
\end{aligned}
$$


which is the same expression as in the case of white noise (Eq. 37). Therefore, the asymptotic behavior at high frequencies of the instantaneous rate modulation is not affected by the presence of temporal correlations in the noise.

\section{References}

Abbott LF, van Vreeswijk C (1993) Asynchronous states in a network of pulse-coupled oscillators. Phys Rev E Stat Phys Plasmas Fluids Relat Interdiscip Topics 48:1483-1490.

Bair W, Koch C (1996) Temporal precision of spike trains in extrastriate cortex of the behaving macaque monkey. Neural Comput 8:1185-1202.

Brunel N (2000) Dynamics of sparsely connected networks of excitatory and inhibitory spiking neurons. J Comput Neurosci 8:183-208.

Brunel N, Hakim V (1999) Fast global oscillations in networks of integrateand-fire neurons with low firing rates. Neural Comput 11:1621-1671.

Brunel N, Chance F, Fourcaud N, Abbott L (2001) Effects of synaptic noise and filtering on the frequency response of spiking neurons. Phys Rev Lett $86: 2186-2189$.

Carandini M, Mechler F, Leonard CS, Movshon JA (1996) Spike train encoding by regular-spiking cells of the visual cortex. J Neurophysiol 76:3425-3441.

Chance F (2000) Modeling cortical dynamics and the responses of neurons in the primary visual cortex. $\mathrm{PhD}$ thesis, Brandeis University.

Ellerkmann RK, Riazanski V, Elger CE, Urban BW, Beck H (2001) Slow recovery from inactivation regulates the availability of voltage-dependent $\mathrm{Na}^{+}$channels in hippocampal granule cells, hilar neurons and basket cells. J Physiol (Lond) 532:385-397.

Ermentrout GB (1996) Type I membranes, phase resetting curves, and synchrony. Neural Computation 8:979-1001.

Ermentrout GB, Kopell N (1986) Parabolic bursting in an excitable system coupled with a slow oscillation. SIAM J Appl Math 46:233-253.

Fleidervish IA, Friedman A, Gutnick MJ (1996) Slow inactivation of $\mathrm{Na}^{+}$ current and slow cumulative spike adaptation in mouse and guinea-pig neocortical neurones in slices. J Physiol (Lond) 493:83-97.

Fourcaud N, Brunel N (2002) Dynamics of firing probability of noisy integrate-and-fire neurons. Neural Comput 14:2057-2110.

Fricker D, Verheugen JAH, Miles R (1999) Cell-attached measurements of the firing threshold of rat hippocampal neurones. J Physiol (Lond) 517:791-804.

Geisler CM, Brunel N, Fourcaud N, Wang X-J (2002) The interplay between intrinsic spiking dynamics and recurrent synaptic interactions in the generation of stochastic network oscillations. Soc Neurosci Abstr 28:276.12.

Gerstner W (2000) Population dynamics of spiking neurons: fast transients, asynchronous states, and locking. Neural Comput 12:43-89.

Green DM, Swets JA (1966) Signal detection theory and psychophysics. New York: Wiley.

Hansel D, Mato G (2001) Existence and stability of persistent states in large neuronal networks. Phys Rev Lett 10:4175-4178.

Hansel D, Mato G (2003) Asynchronous states and the emergence of synchrony in large networks of interacting excitatory and inhibitory neurons. Neural Comput 15:1-56.
Hansel D, van Vreeswijk C (2002) How noise contributes to contrast invariance of orientation tuning in cat visual cortex. J Neurosci 22:5118-5128.

Haskell E, Nykamp DQ, Tranchina D (2001) Population density methods for large-scale modelling of neuronal networks with realistic synaptic kinetics: cutting the dimension down to size. Network 12:141-174.

Hodgkin AL, Huxley AF (1952) A quantitative description of membrane current and its application to conductance and excitation in nerve. J Physiol (Lond) 117:500-544.

Honeycutt RL (1992) Stochastic Runge-Kutta algorithms. I. White noise. Phys Rev A 45:600-603.

Knight BW (1972a) Dynamics of encoding in a population of neurons. J Gen Physiol 59:734-766.

Knight BW (1972b) The relationship between the firing rate of a single neuron and the level of activity in a population of neurons. J Gen Physiol 59:767-778.

Knight BW, Omurtag A, Sirovich L (2000) The approach of a neuron population firing rate to a new equilibrium: an exact theoretical result. Neural Comput 12:1045-1055.

Martina M, Jonas P (1997) Functional differences in $\mathrm{Na}^{+}$channel gating between fast-spiking interneurons and principal neurones of rat hippocampus. J Physiol (Lond) 505:593-603.

Mazurek ME, Shadlen MN (2002) Limits to the temporal fidelity of cortical spike rate signals. Nat Neurosci 5:463-471.

McCormick D, Connors B, Lighthall J, Prince D (1985) Comparative electrophysiology of pyramidal and sparsely spiny stellate neurons in the neocortex. J Neurophysiol 54:782-806.

Nowak L, Sanchez-Vives M, McCormick D (1997) Influence of low and high frequency inputs on spike timing in visual cortical neurons. Cereb Cortex 7:487-501.

Nykamp DQ, Tranchina D (2000) A population density approach that facilitates large-scale modeling of neural networks: analysis and an application to orientation tuning. J Comp Neurosci 8:19-30.

Powers RK, Binder MD (2001) Input-output functions of mammalian motoneurons. Rev Physiol Biochem Pharmacol 143:137-263.

Ris L, Hachemaoui M, Vibert N, Godaux E, Vidal PP, Moore LE (2001) Resonance of spike discharge modulation in neurons of the guinea pig medial vestibular nucleus. J Neurophysiol 86:703-716.

Risken H (1984) The Fokker-Planck equation: methods of solution and applications. Berlin: Springer.

Sekirnjak C, du Lac S (2002) Intrinsic firing dynamics of vestibular nucleus neurons. J Neurosci 22:2083-2095.

Treves A (1993) Mean-field analysis of neuronal spike dynamics. Network 4:259-284.

Tuckwell HC (1988) Introduction to theoretical neurobiology. Cambridge: Cambridge UP.

van Rossum MCW, Turrigiano GG, Nelson SB (2002) Fast propagation of firing rates through layered networks of noisy neurons. J Neurosci 22:1956-1966.

Wang X-J, Buzsáki G (1996) Gamma oscillation by synaptic inhibition in a hippocampal interneuronal network model. J Neurosci 16:6402-6413. 